ARTICLE

\title{
Calreticulin del52 and ins5 knock-in mice recapitulate different myeloproliferative phenotypes observed in patients with MPN
}

\author{
Camélia Benlabiod ${ }^{1,2,3}$, Maira da Costa Cacemiro ${ }^{1,2,3,4}$, Audrey Nédélec ${ }^{5,6}$, Valérie Edmond $d^{1,2,3}$, \\ Delphine Muller 1,2,3, Philippe Rameau ${ }^{7}$, Laure Touchard ${ }^{8}$, Patrick Gonin (1) ${ }^{8}$, Stefan N. Constantinescu (1) ${ }^{5,6}$, \\ Hana Raslova1,2,3, Jean-Luc Villeval ${ }^{1,2,3}$, William Vainchenker (10) ${ }^{1,2,3}$, Isabelle Plo ${ }^{1,2,3,9}$ \& Caroline Marty (1) ${ }^{1,2,3,9 凶}$
}

Somatic mutations in the calreticulin (CALR) gene are associated with approximately $30 \%$ of essential thrombocythemia (ET) and primary myelofibrosis (PMF). CALR mutations, including the two most frequent 52 bp deletion (del52) and 5 bp insertion (ins5), induce a frameshift to the same alternative reading frame generating new C-terminal tails. In patients, del52 and ins 5 induce two phenotypically distinct myeloproliferative neoplasms (MPNs). They are equally found in $\mathrm{ET}$, but del52 is more frequent in PMF. We generated heterozygous and homozygous conditional inducible knock-in (KI) mice expressing a chimeric murine CALR del52 or ins 5 with the human mutated $\mathrm{C}$-terminal tail to investigate their pathogenic effects on hematopoiesis. Del52 induces greater phenotypic changes than ins5 including thrombocytosis, leukocytosis, splenomegaly, bone marrow hypocellularity, megakaryocytic lineage amplification, expansion and competitive advantage of the hematopoietic stem cell compartment. Homozygosity amplifies these features, suggesting a distinct contribution of homozygous clones to human MPNs. Moreover, homozygous del52 KI mice display features of a penetrant myelofibrosis-like disorder with extramedullary hematopoiesis linked to splenomegaly, megakaryocyte hyperplasia and the presence of reticulin fibers. Overall, modeling del52 and ins 5 mutations in mice successfully recapitulates the differences in phenotypes observed in patients.

\footnotetext{
${ }^{1}$ INSERM, UMR 1287, Gustave Roussy, Villejuif, France. ${ }^{2}$ Université Paris-Saclay, UMR 1287, Gustave Roussy, Villejuif, France. ${ }^{3}$ Gustave Roussy, UMR 1287, Villejuif, France. ${ }^{4}$ Department of Clinical Analysis, Toxicology and Food Sciences, School of Pharmaceutical Sciences of Ribeirão Preto, University of São Paulo-USP, Ribeirão Preto, São Paulo, Brazil. ${ }^{5}$ Ludwig Institute for Cancer Research, Brussels, Belgium. ${ }^{6}$ de Duve Institute, Walloon Excellence in Life Sciences and Biotechnology (WELBIO), Université catholique de Louvain, Brussels, Belgium. ${ }^{7}$ Integrated Biology Core Facility, Gustave Roussy, Villejuif, France. ${ }^{8}$ Preclinical Research Plateform, Unité Mixte de Service AMMICA 3655/US 23, Gustave Roussy, Villejuif, France. ${ }^{9}$ These authors contributed equally: Isabelle Plo, Caroline Marty. ${ }^{凶}$ email: caroline.marty@gustaveroussy.fr
} 
$\mathrm{M}$ yeloproliferative neoplasms (MPNs) are driven by the acquisition of somatic mutations in the hematopoietic stem cell (HSC) compartment leading to the constitutive activation of the JAK-STAT signaling pathway. Essential thrombocythemia (ET) and primary myelofibrosis (PMF) are two disorders of the megakaryocytic lineage defined by an increased level of circulating platelets in ET and by megakaryocytic hyperplasia/ dysplasia accompanied by the development of fibrosis in the bone marrow (BM) in PMF. PMF can display several other clinical features, including leukocytosis, splenomegaly, due to extramedullary hematopoiesis and anemia that may progress into pancytopenia. ET can evolve into a pre-fibrotic stage and overt secondary myelofibrosis (MF). The main driver mutations of ET and PMF are $J A K 2^{\mathrm{V} 617 \mathrm{~F}}$, mutations in the exon 9 of the calreticulin (CALR) gene, and in the thrombopoietin (TPO) receptor $M P L$ gene $^{1-4}$. Several CALR mutations have been reported and all induce $\mathrm{a}+1$-base pair (bp) frameshift to an alternative reading frame leading to the replacement of the negatively-charged Cterminal tail to a new positively charged sequence with 34 residues common to all mutants. It was shown that CALR mutants specifically bind to and constitutively activate MPL, explaining the megakaryocyte (MK) phenotype of the CALR-mutated MPNs ${ }^{5,6}$.

The two most frequent $C A L R$ mutations are a 52-bp deletion (del52) or type 1 and a 5-bp insertion (ins5) or type 2. The frequency of the two types of mutations is close in ET while del52 and rarer type 1-like mutations are largely predominant in MF, thus, del52 and ins 5 define two phenotypically distinct MPNs in human ${ }^{7}$. Retroviral (RV) modeling in mice has shown that the two types of human CALR mutations induce a MPL-dependent phenotype mimicking human disorders, but this approach presents pitfalls such as the ectopic over-expression of CALR mutants compared to endogenous wild-type (wt) $\mathrm{CALR}^{8,9}$. Several other models have been published including a human del52 transgenic (TG) mouse ${ }^{10}$, CRISPR-Cas9-mediated both murine del19 and murine del52 $2^{11}$, and murine del61 knock-in (KI) mice ${ }^{12}$ as well as an inducible conditional KI expressing murine CALR with the human del52-mutated end tail ${ }^{13}$. Yet, none of these studies directly compare the effects of in vivo physiopathological expression of the two most frequent types of CALR mutations on hematopoiesis, nor do they specifically address the disease outcomes of ins 5 or type 2-like mutant expression in mice.

Understanding the basis of the phenotypic differences induced by type 1 and type 2 mutations is of deep interest as their impact on the evolution of the disease and thus patient prognosis differ. In the present work, we conducted a direct comparison of the effects of heterozygous and homozygous physiopathological expression of del52 and ins5 mutants on hematopoiesis using inducible conditional KI mice. We chose to express chimeric mouse/human proteins based on the fact that mutated C-terminal tails of the two species display different amino acid composition and we thus generated these del52 and ins5 KI mice before the recent demonstration that the human and murine mutated C-terminal tails have close oncogenic properties ${ }^{11,14}$. Expression of del52 induces a stronger phenotype including thrombocytosis, leukocytosis, megakaryocytic and progenitor amplification as well as a penetrant fibrosis in spleen compared to ins5. Moreover, these phenotypes are both amplified by homozygosity suggesting a possible distinct contribution of the homozygous clone to the development and the extent of the human disorders. Thus, our KI mice recapitulate the human MPNs and provide powerful preclinical models to decipher the molecular bases of the differences between del52 and ins 5 diseases.

\section{Results}

Generation of KI mice for CALR del52 and CALR ins5. We used the FLEx strategy to generate conditional heterozygous mice carrying a floxed construct fusion of mouse Calr exons 8 and 9 followed with wild-type mouse Calr exon 8 and a modified exon 9 encompassing either the murine deletion of 52-bp (CALR del52) or a 5-bp insertion (CALR ins5) analogous to human mutations and the human mutated $\mathrm{C}$-terminal sequence. To generate $\mathrm{KI}$ mice, floxed mice were crossed with SCL-CreER ${ }^{T}$ TG animals expressing a tamoxifen-inducible Cre. Thus, after induction of the KI allele expression by Cre recombinase these mice express heterozygous mouse CALR with human mutated C-terminal tail from its endogenous promoter (Fig. 1a, b). We purified BM lineage-negative $\left(\mathrm{Lin}^{-}\right)$cell population for each $\mathrm{KI}$ model and measured CALR protein levels by Western blot (Fig. 1c). Using an antibody (Ab) (CALR-tot) that recognizes both CALR wt and mutants we mostly detected doublet bands at the expected CALR protein size that might reflect the presence of a cleaved product and/or protein post-translational modifications. Identical result was obtained using a different Ab clone (Supplementary Fig. 1a) and expression levels of three independent experiments were quantified (Supplementary Fig. 1b). We observed no difference in CALR expression between wt $(+/+)$ and heterozygous $+/$ del52 and + /ins 5 cells. However, we noticed that in the absence of endogenous CALR wt (homozygous del52/del52 and ins5/ins5 cells) the amount of CALR mutant protein in cell lysate was lower (only significant with $1.8-$ fold less CALR del52 detected in del52/ del52 compared to $+/+$ cells) than in both wt and heterozygous cells. Using an Ab specifically directed against the mutated Cterminal tail (CALR-Cter) that does not detect endogenous CALR wt, there seems to be less CALR del52 than CALR ins5 in the homozygous cells, in accordance with the difference of protein levels revealed with the CALR-tot Ab. Surprisingly, we barely observed any band in the heterozygous cell lysates.

KI mouse phenotypes depend on the mutation and zygosity. Both del52 and ins 5 KI mice developed a rapid thrombocytosis after induction by tamoxifen compared to wt $(+/+)$ mice, but at higher levels in the presence of del52 than in the presence of ins5 (Fig. 2a, b, Supplementary Fig. 1c). Moreover, we observed that the thrombocytosis was more elevated in homozygous compared to heterozygous context. Both del52 and ins5 KI mice, especially in the homozygous context, also developed a significant leukocytosis (Fig. 2c, d). Compared to wt controls, hemoglobin levels were normal in all KI models except significantly lower in homozygous del52 mice and starting to decrease in 7-month-old homozygous ins 5 mice (Fig. 2e, f).

Leukocytosis developed by KI mice, especially in the homozygous setting, was unexpected from CALR-mutated patient studies and was due to a general increase in the different white blood cell (WBC) populations (Supplementary Fig. 2a, b). There was a significant increase in neutrophils, lymphoid cells, and eosinophils. Monocytosis was higher in presence of del52 than in the presence of ins5, while basophilia was more important in presence of ins 5 than del52 (Supplementary Fig. 2a, b). The del52 mutation was shown to lead to a general increase of various $\mathrm{T}$ lymphocyte sub-populations (Supplementary Fig. 3a, b).

Effect of the CALR mutations on the megakaryocytic lineage. We studied the effect of del52 and ins 5 on the MK lineage, and observed a TPO-independent MK progenitor (CFU-MK) growth in heterozygous and homozygous del52 and ins5 $\mathrm{KI}$ mice, as stimulation by TPO induced only a significant increase of CFUMK frequency in $+/+$ mice (Fig. 3a). Moreover, we measured the frequencies of $\mathrm{MK}$ progenitors (MkPs) and $\mathrm{MKs}\left(\mathrm{CD} 41^{+} \mathrm{CD} 42^{+}\right)$ in BM (Fig. 3b) and spleen (Fig. 3c) of KI mice by flow cytometry. We observed increased MkPs and MKs in del52/del52 KI mice and to a lower extent for MkPs in ins5/ins5 KI mice. Accordingly, 
a

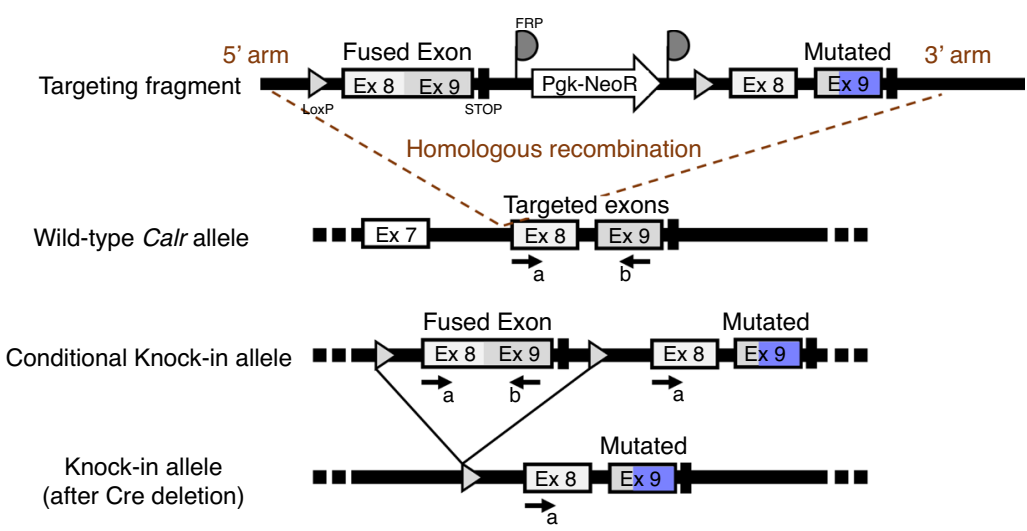

Mouse Calr wt

Exon 8

Exon 9

5'-gtcaagtccgggacaatctttgacaatttcctcatcaccaatgatgaggcctatgcagaggagtttggcaatgagacgtggggtgttaccaaggctgcagagaagcag atgaaggacaagcaggatgaggagcagaggcttaaggaagaagaagaggacaagaagcgtaaagaggaagaagaagctgaggataaagaggatgatgatgac agagatgaagatgaggacgaagaagatgagaaggaggaagatgaggaagaatcccctggccaagccaaggatgagctgtag - $3^{\prime}$

Mouse/human CALR ins5

5'-gtcaagtccgggacaatcttgacaattcctcatcaccaatgatgaggcctatgcagaggagtttggcaatgagacgtggggtgttaccaaggctgcagagaagcag atgaaggacaagcaggatgaggagcagaggcttaaggaagaagaagaggacaagaagcgtaaagaggaagaagaagctgaggataaTTGTCGGAGGA TGATGAGGACAAAGATGAGGATGAGGAGGATGAGGAGGACAAGGAGGAAGATGAGGAGGAAGATGTCCCCGGCCA GGCCAAGGACGAGCTGTAGAGAGGCCTGCCTCCAGGGCTGGACTGAGGCCTGA-3

Mouse/human CALR del52

5'-gtcaagtccgggacaatctttgacaatttcctcatcaccaatgatgaggcctatgcagaggagtttggcaatgagacgtggggtgttaccaaggctgcagagaagcag atgaaggacaagcaggatgaggagcagaggACAAGGAGGATGATGAGGACAAAGATGAGGATGAGGAGGATGAGGAGGACAAG GAGGAAGATGAGGAGGAAGATGTCCCCGGCCAGGCCAAGGACGAGCTGTAGAGAGGCCTGCCTCCAGGGCTGGA CTGAGGCCTGA-3'

b

Mouse CALR wt

--AAEKQMKDKQDEEQRLKEEEEDKKRKEEEEAEDKEDDDDRDEDEDEEDEKEEDEEESPGQAKDEL

Mouse/human CALR ins5

--AAEKQMKDKQDEEQRLKEEEEDKKRKEEEEAEDNCRRMMRTKMRMRRMRRTRRKMRRKMSPARPRTSCREACLQGWTEA

Mouse/human CALR del52

--AAEKQMKDKQDEEQRTRRMMRTKMRMRRMRRTRRKMRRKMSPARPRTSCREACLQGWTEA

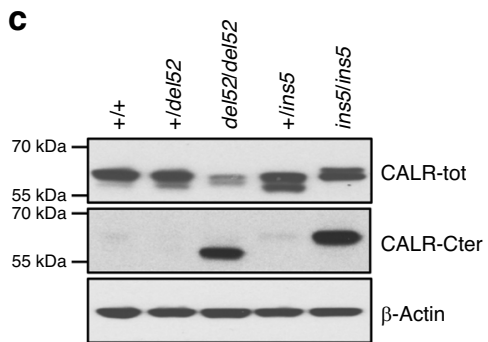

Fig. 1 Conditional CALR del52 and CALR ins5 KI mouse models. a Schematic representation of the wt and conditional KI alleles obtained after crossing the chimeras with flippase TG mice. KI allele was obtained after crossing mice with SCL-CreER ${ }^{\top}$ TG mice and induction of Cre-mediated deletion of the wt fused exon 8 and 9 by tamoxifen, arrows are LoxP sites. The heterozygous and homozygous status of conditional KI mice was verified by DNA genotyping using a and b primers. A similar strategy was used to genotype conditional ins 5 and del52 KI mice. Sequence of mouse Calr (lower cases) exon (Ex) 8 (italic gray) and Ex 9 (black) was depicted with the 52-bp (red) deleted in the mouse/human CALR del52 chimera and the green arrow pointing to the position of the 5-bp insertion (TTGTC in green) in CALR ins5. Human sequence is depicted in upper cases and the purple sequence encodes the human mutated Cterminal tail. b Protein sequences of mouse CALR wt Ex 9, and CALR del52 and CALR ins5 mutated Ex 9. In black is mouse sequence and in purple human sequence with the common mutated C-terminus underlined. $\mathbf{c}$ Lin $^{-}$cells isolated from BM of wt $(+/+)$, heterozygous $(+/$del52 or $+/$ins 5$)$ and homozygous (del52/del52 and ins5/ins5) CALR-mutated KI mice were analyzed by Western blot for expression of CALR using an Ab directed against the Nterminus, common to both wt and mutant CALR (CALR-tot, Cell Signaling) and an Ab specific of the mutated C-terminal tail (CALR-Cter). B-Actin was used as loading control. Blots shown are representative of at least three independent experiments. Source data are provided as a Source data file.

immuno-histological staining for von Willebrand factor (vWF) confirmed MK hyperplasia in BM (Fig. 3d) and spleen (Supplementary Fig. 4a) of homozygous del52 mice while homozygous ins 5 mice mostly presented an apparent increase in the MK size and the number of MKs but only in the spleen. Milder phenotype was found with heterozygous del52. Independently of zygosity, all KI mice except +/ins5 animals, displayed giant MKs with polylobulated nuclei suggesting increased modal ploidy. Indeed, in vivo MKs achieved a significantly higher zygosity-dependent ploidy in del52 KI mice than in wt littermates (Fig. 3e). The mean ploidy of ins5/ins5 MKs was identical to del52/del52 MKs, both displaying also a modal ploidy of $32 \mathrm{~N}$ compared to $16 \mathrm{~N}$ in wt littermates (Supplementary Fig. 4b), while there was no marked difference between ploidy of $+/$ ins 5 and wt MKs with the same 

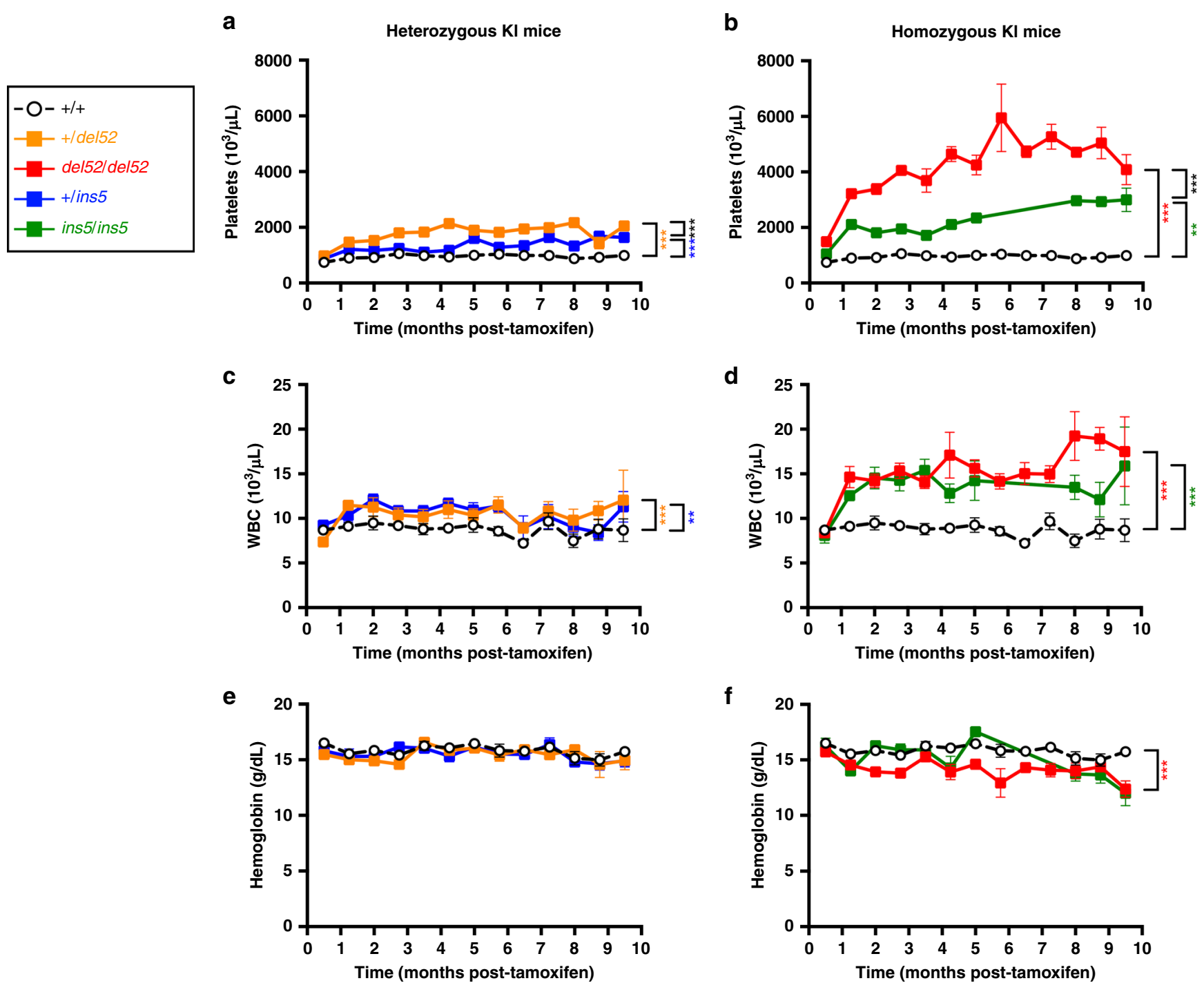

Fig. 2 Severity of KI mouse phenotype depends on the mutation and the zygosity. Blood parameters including $\mathbf{a}, \mathbf{b}$ platelets, $\mathbf{c}, \mathbf{d}$ WBC counts and e, f hemoglobin levels were determined in wt $+/+$ (open circles with connecting black dashed line, $n=16,18,15,11,11,8,13,7,14,11,5,6,4$ for platelets, $n=34,25,26,25,19,18,17,12,19,13,10,13,7$ for WBC and $n=36,26,28,25,21,19,20,11,19,11,9,14,9$ for hemoglobin), heterozygous $+/$ del52 (orange squares and solid line, $n=18,20,15,12,10,7,11,7,12,12,8,5,10$ for platelets, $n=18,19,15,13,11,7,11,7,14,11,7,5,3$ for WBC and $n=19,20,16,13,11,8$, $11,7,13,9,7,5,3$ for hemoglobin) and +/ins5 (blue squares and solid line, $n=20,7,13,18,11,13,10,5,4,4,5,8,7$ for platelets, $n=21,8,12,19,11,12,10$, $5,5,4,5,8,9$ for WBC and $n=20,7,12,18,11,13,13,5,5,4,5,8,7$ for hemoglobin) and homozygous del52/del52 (red squares and solid line, $n=14,14$, $10,7,6,3,8,3,7,5,3,3,4$ for platelets, $n=14,14,10,8,7,5,8,2,7,5,4,3,4$ for WBC and $n=16,16,10,7,7,5,8,3,7,4,3,3,4$ for hemoglobin) and ins5/ins5 (green squares and solid line, $n=6,1,6,8,9,7,5,6,5,5$ for platelets, $n=8,1,7,9,9,6,6,6,6,4$ for WBC and $n=6,1,7,9,9,8,5,6,5,6$ for hemoglobin) mice after tamoxifen induction. $N$ represents the number of individual mouse and varies depending on time of sampling during the course of the experiment, as indicated in the Source data file. Data are the means \pm SEM. Significance was determined using ANOVA with Tukey's multiple comparison test: ${ }^{\star \star} p=0.001,{ }^{\star \star \star} p \leq 0.0001(\mathbf{a}, \mathbf{b}) ;{ }^{\star \star} p \leq 0.001,{ }^{\star \star \star} p=0.0007(\mathbf{c}) ;{ }^{\star \star \star} p<0.0001(\mathbf{d}) ;{ }^{\star \star \star} p=0.0008$ (f). Source data are provided as a Source data file.

modal ploidy. However, in comparison to wt MKs, the $8 \mathrm{~N}$ peak decreased in favor of the $32 \mathrm{~N}$ peak in + /ins 5 MKs. These data suggest that del52 induces a stronger amplification of MK progenitor cells compared to ins5 (increase in MkP and MK numbers) while both mutations affect the late stages of megakaryopoiesis (increase in MK ploidy), indicating that CALR ins5 seems less effective than CALR del52.

CALR del52 KI mice develop a myelofibrosis-like disease. Mice were analyzed after 10 months of tamoxifen induction. There was a significant decrease in BM cellularity of del52/del52 mice combined with an increase in spleen weight (Fig. 4a, b). There was barely a difference in the $+/$ del52, ins5/ins5, and +/ins 5 mice compared to wt controls. In the del52/del52 mice, the 4-fold increase in the frequency of giant MKs in the BM (Fig. 3) may partially explain the decrease in BM cellularity. Moreover, in the BM of del52/del52 mice, we observed a decrease in the frequencies of both erythroid progenitors (BFU-E) and erythroblasts (Ter-119+) (Supplementary Fig. 5a) with no noticeable change in granulo-monocytic progenitors (CFU-GM) and granulocyte precursors $\left(\mathrm{Macl}^{+} \mathrm{Gr}^{+}\right.$) (Supplementary Fig. $5 \mathrm{c}$ ) or the lymphoid cells $\left(\mathrm{CD}^{+}\right.$and $\left.\mathrm{B}^{+} 20^{+}\right)$ (Supplementary Fig. 5e). Histological examination by silver staining showed a very mild MF in del52/del52 mice but no significant development of reticulin fiber network in the BM of any other KI mice (Fig. 4c, BM, reticulin silver (RET) staining), but we observed a neo-osteogenesis only in rare cases of del52/del52 mice. 

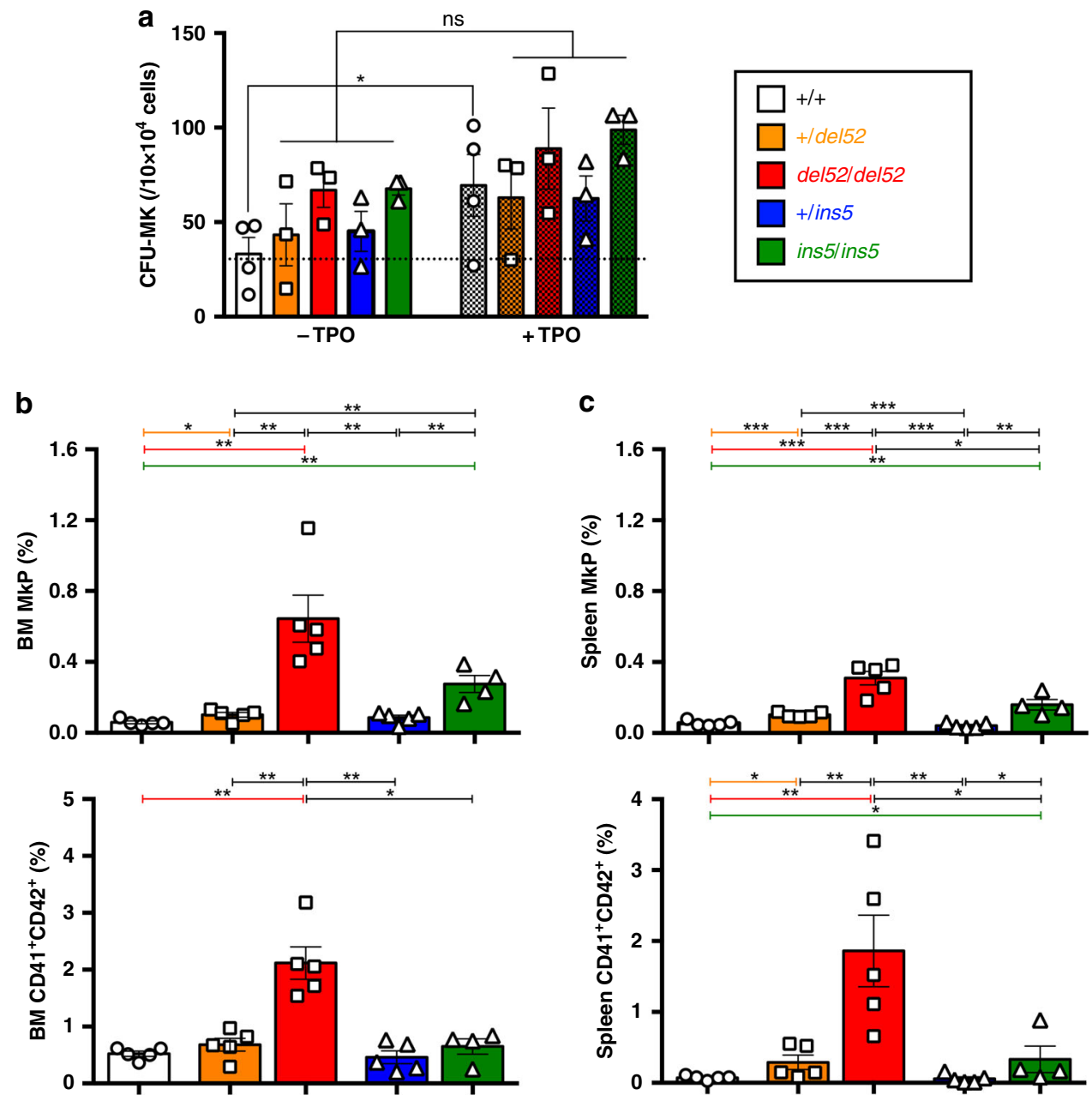

d

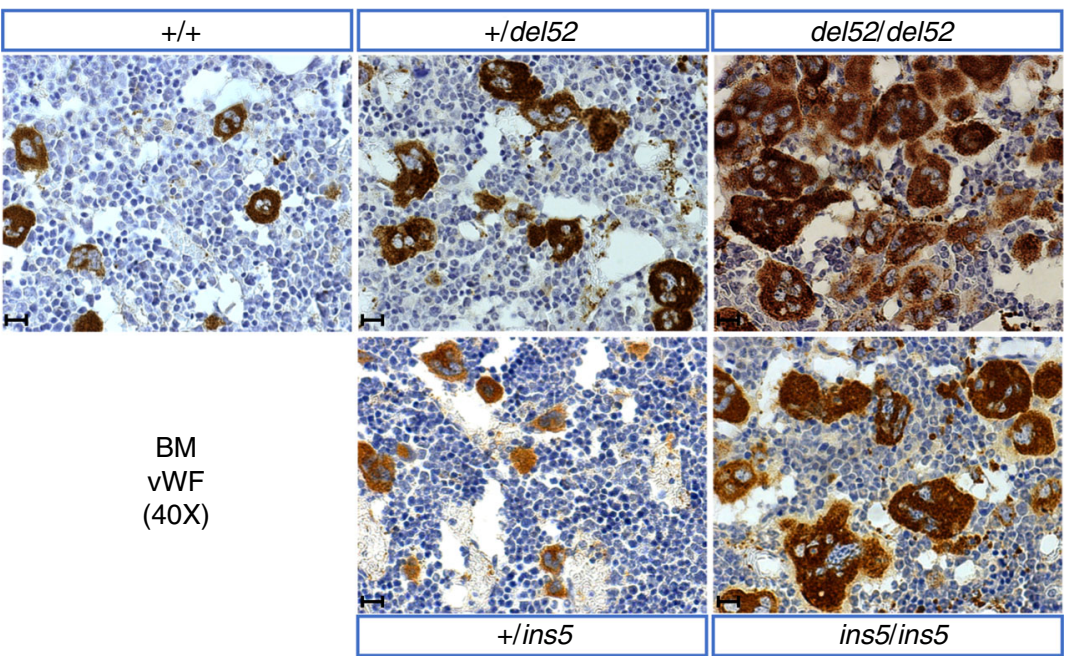

e

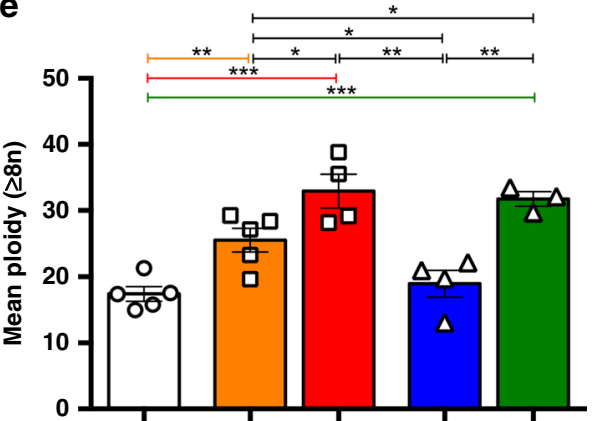

The unexpected quasi-absence of fibrosis in BM, particularly of homozygous del52 KI mice that present an important MK hyperplasia confirmed on hematoxylin/eosin/safran (HES) staining (Fig. 4c), prompted us to explore fibrosis in spleen. Histology revealed the presence of a thicker reticulin fiber network in homozygous del52 compared to wt spleen, and in a less penetrant fashion in homozygous ins5 KI mice (Fig. 4c, Spleen, RET). Spleen structure was partially disorganized in the +/del52 and ins5/ins5 KI mice and, in del52/del52 KI mice, white pulp territories were almost lost (Supplementary Fig. 6), attributable to a strong MK hyperplasia (Fig. 4c, Spleen, HES) and an increase in RBCs (Fig. 4d) that was confirmed by semisolid cultures and flow cytometry analysis at the progenitor and erythroblast levels (Supplementary Fig. 5b). An increase in granulo-monocytic lineage cells was also observed only in del52/ del52 KI spleens (Supplementary Fig. 5d), explaining the higher spleen weights of these mice compared to the three other phenotypes and the wt littermates (Fig. 4b). Disorganization of 
Fig. 3 Amplification of the megakaryocytic lineage in KI mice. a Frequencies of BM megakaryocytic progenitors (CFU-MKs) with or without TPO of $\geq 10$ months post-tamoxifen KI mice were represented as means \pm SEM $(n=4$ individual $+/+$ mice and $n=3$ mice for heterozygous and homozygous genotypes). In the figure, white is used for $+/+$, orange for $+/$ del 52 , red for del $52 /$ del 52 , blue for $+/$ ins 5 and green for ins $5 /$ ins 5 genotypes. Significance of +TPO (hatched histograms) compared to the - TPO (solid histograms) condition was assessed using a two-sided parametric paired $t$-test $\left({ }^{\star} p=0.0192 ; n s\right.$ not significant). b, c Frequencies of megakaryocytic progenitors (MkPs, $\left.\mathrm{Lin}^{-} \mathrm{C}-\mathrm{Kit}{ }^{+} \mathrm{Sca}-{ }^{-} \mathrm{CD} 150^{+} \mathrm{CD} 41^{+}\right)$and $\mathrm{MKs}\left(\mathrm{CD} 41^{+} \mathrm{CD} 42^{+}\right)$in $\mathbf{b} \mathrm{BM}$ and $\mathbf{c}$ spleen cells from $+/+,+/$ del52, and del52/del52 compared to $+/$ ins 5 and ins $5 /$ ins 5 mice $\geq 10$ months after tamoxifen induction were expressed as means \pm SEM ( $n=5$ individual mice except $n=4$ ins5/ins 5 mice). Significance for MkPs was calculated with a two-sided parametric $t$-test and for MKs with a two-sided nonparametric Mann-Whitney test. In $\mathbf{b},{ }^{\star} p=0.023 ;{ }^{\star \star} p=0.0023$ (+/+ vs. del52/del52), 0.0016 (+/+ vs. ins5/ins5), 0.0036 (+/del52 vs. del52/del52), 0.0063 (+/del52 vs. ins5/ins5), 0.0031 (del52/del52 vs. $+/$ ins5), 0.004 ( + /ins5 vs. ins5/ins5) in MkP analysis and ${ }^{\star} p=0.0159 ;{ }^{* \star} p=0.0079$ in CD $41+C D 42+$ analysis. In ${ }^{*}{ }^{\star} p=0.0213$ (del52/del52 vs. ins5/ins5); ${ }^{\star \star} p=0.0058\left(+/+\right.$ vs. ins5/ins5), 0.003 ( + /ins5 vs. ins5/ins5); ${ }^{\star \star \star} p=0.0007(+/+$ vs. $+/$ del52), 0.0002 (+/+ vs. del52/del52), 0.0008 (+/del52 vs. del52/del52), <0.0001 (+/del52 vs. +/ins5), 0.0001 (del52/del52 vs. $+/$ ins5) in MkP analysis and ${ }^{*} p=0.0159$ (+/+ vs. +/del52), 0.0238 (+/+ vs. ins5/ins5), 0.0317 (del52/de/52 or +/ins5 vs. ins5/ins5); ${ }^{* *} p=0.0079$ in CD41 ${ }^{+}$CD42 ${ }^{+}$ analysis. d BM MKs were immunostained for vWF. Images were obtained using a DM2000 Leica microscope and a DFC300FX Leica camera with Leica Application Suite v.2.5,OR1 acquisition software ( $\times 40$ magnification). Scale bars represent $50 \mu \mathrm{m}$. Similar results were obtained with analyzing at least three independent mice for each genotype. e Mean ploidy of $\geq 8 \mathrm{~N}$ MKs from $\geq 10$ months post-tamoxifen KI mice was expressed as means \pm SEM ( $n=5$ individual $+/+$ and $+/$ del52 mice, $n=4$ del52/del52 and $+/$ ins 5 mice and $n=3$ ins $5 /$ ins 5 mice). Statistical significance was determined using a two-sided parametric $t$-test: ${ }^{\star} p=0.0453$ (+/del52 vs. del52/del52), 0.0454 (+/del52 vs. $+/$ ins5), $0.0498\left(+/\right.$ del52 vs. ins5/ins5); ${ }^{\star \star} p=0.0047(+/+$ vs. $+/$ del52), 0.0052 (del52/del52 vs. +/ins5), 0.0042 (+/ins5 vs. ins5/ins5); ${ }^{\star \star \star} p=0.0005(+/+$ vs. del52/del52), 0.00001 (+/+vs. ins5/ins5). Source data are provided as a Source data file.

white pulp in spleens of homozygous del52 and, to a lesser extent, ins5 animals was accompanied with a decrease in the frequencies of lymphocytes, but their absolute numbers increased non-significantly relative to splenomegaly (Supplementary Fig. 5f). These results show that del52/del52 in contrast to ins5/ins 5 mice present early signs of myelofibrosis characterized by a BM cellularity decrease, splenomegaly and extramedullary hematopoiesis even if distinct reticulin fibers could not be significantly detected in the BM in contrast to the spleen.

Phenotype severity depends on MPL/JAK2/STAT activation. Since fibrosis was milder in BM compared to spleen, we tested whether CALR del52 might induce a weaker signaling in BM compared to spleen MKs by phosphoflow. CALR del52 induced the constitutive phosphorylation of STAT5 in homozygous BM MKs compared to wt $(+/+)$ littermate BM MKs that was dependent on JAK2 as level returned to basal with the JAK1/2 inhibitor ruxolitinib (Supplementary Fig. 7a). Interestingly, TPO stimulation of del52/del52 MKs increased the level of phosphorylated STAT5 suggesting that at least a certain amount of MPL was accessible for TPO binding in presence of CALR del52. However, we did not observe any obvious difference between del52-induced phosphorylation of STAT5 in BM compared to spleen MKs. In our mice, the KI allele encodes the murine (mu) CALR ins5 or del52 with human (hu) mutated C-terminal tail. In order to determine the efficacy of these chimeric (chim) CALR mutants, compared to hu CALR del52 and ins5, to activate the mu MPL, we measured STAT5 activation by luciferase assay in $\gamma 2 \mathrm{~A}$ cells. In this system, mu MPL was indistinctively and spontaneously (-TPO) activated by chim CALR del52, chim CALR ins5, hu CALR del52 and hu CALR ins5 (Fig. 5a). As previously shown for the hu CALR mutants, the chim CALR mutants were also less efficient in constitutively activating mu MPL compared to hu MPL. As expected, stimulation of $\mathrm{mu} / \mathrm{hu}$ MPL with TPO abolished the differences in STAT5 activation in presence of any of the CALR mutants and CALR wt. In order to verify whether the mild MF phenotype developed by del52/del52 KI mice was due to sub-optimal stimulation of mu MPL by the chim CALR del52 protein, we treated 1 month post-induction homozygous KI mice with the MPL agonist romiplostim, that was previously reported to induce MF in wt mice ${ }^{15}$. The romiplostim-treated homozygous del52 KI mice developed a significant BM hypocellularity and splenomegaly compared to treated wt littermates (Fig. 5b) accompanied with an increase in platelets, WBC, and slight anemia (Supplementary Fig. 7b) possibly indicating the development of MF. Romiplostim treatment induced MK hyperplasia revealed by vWF immunostaining and fibrosis with the presence of black reticulin fibers in both BM and spleen of wt and, to a stronger extent, of del52/del52 mice (Fig. 5c). Taken together, this suggests that the del52/del52 KI mice develop a fibrosis in both BM and spleen linked to a constitutive stimulation of mu MPL, but mild as this activation is weak.

Amplification of the HSC compartment in KI mice. The study of the different cell compartments suggested that del52 may provide a stronger advantage to $\mathrm{HSC}\left(\mathrm{Lin}^{-} \mathrm{Sca}-1^{+} \mathrm{c}-\mathrm{Kit}^{+} \mathrm{CD} 48^{-}\right.$ $\mathrm{CD} 150^{+}$, called SLAM) than ins5. In homozygous KI mice after 10 months post-induction, there was a significant amplification of HSC in both BM and spleen that was stronger in presence of del52 compared to ins5 (Fig. 6a and Supplementary Fig. 8). An increasing trend was observed in $+/$ del 52 mice while $+/$ ins 5 was very similar to controls. To decipher the effect of both mutations in the HSC compartment, we performed a competitive BM transplantation with increasing percentages of non-induced homozygous del52 or homozygous ins 5 cells with wt $\mathrm{GFP}^{+}$cells into lethally irradiated recipient mice (Fig. 6b). One month after transplantation KI expression was induced by tamoxifen. Del52engrafted mice developed a higher dose-dependent thrombocytosis (Fig. 6c) and leukocytosis (Fig. 6d) than ins5-engrafted mice and no change in hemoglobin (Supplementary Fig. 9). After 4 months, thrombocytosis developed by mice engrafted with $100 \%$ and $75 \%$ of mutated cells reached similar levels than the respective age-related (4-5 months post-induction) native del52/ del52 and ins5/ins5 mice (Fig. 2b). In contrast to del52-engrafted mice, there was no obvious development of a disease at 4 months with $<50 \%$ of ins5-engrafted cells. Evolution of mutated cells was followed in blood platelets, granulocytes and erythrocytes. The homozygous del52 BM cells were able to outcompete wt BM cells from as low as an initial 10\% competitive engraftment, reaching $100 \%$ at 4 months with a delay in the erythrocytes likely due to the longer half-life of these cells (Fig. 7a). In contrast, competition by ins $5 \mathrm{BM}$ cells was slower especially when $<50 \%$ of mutated cells were initially engrafted (Fig. $7 \mathrm{~b}$ ) suggesting that del52 provides a stronger selective advantage than ins 5 to the HSC. Especially, percentages of ins5-mutated cells engrafted at $10 \%$ and $25 \%$ followed over a 4-month period after KI induction were visibly below values for similar amounts of engrafted del52-mutated 


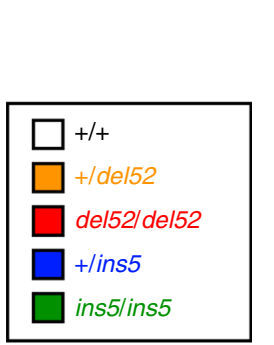

a

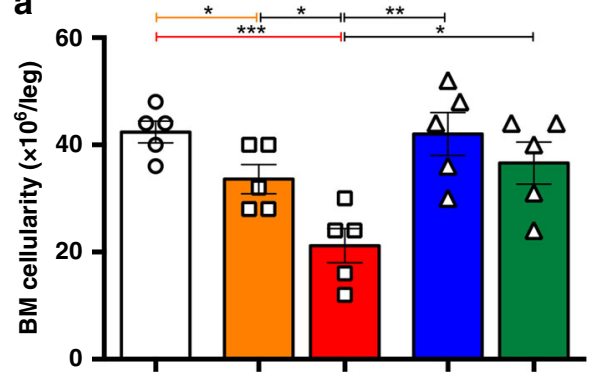

b

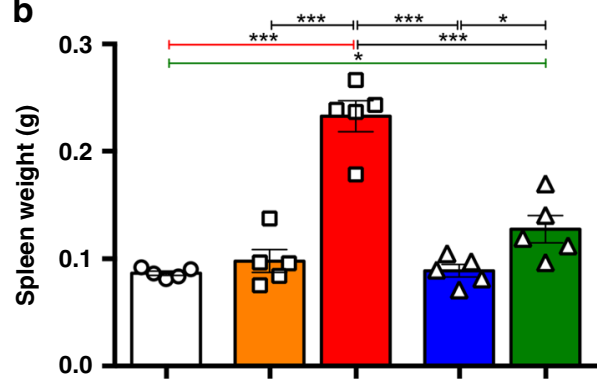

C

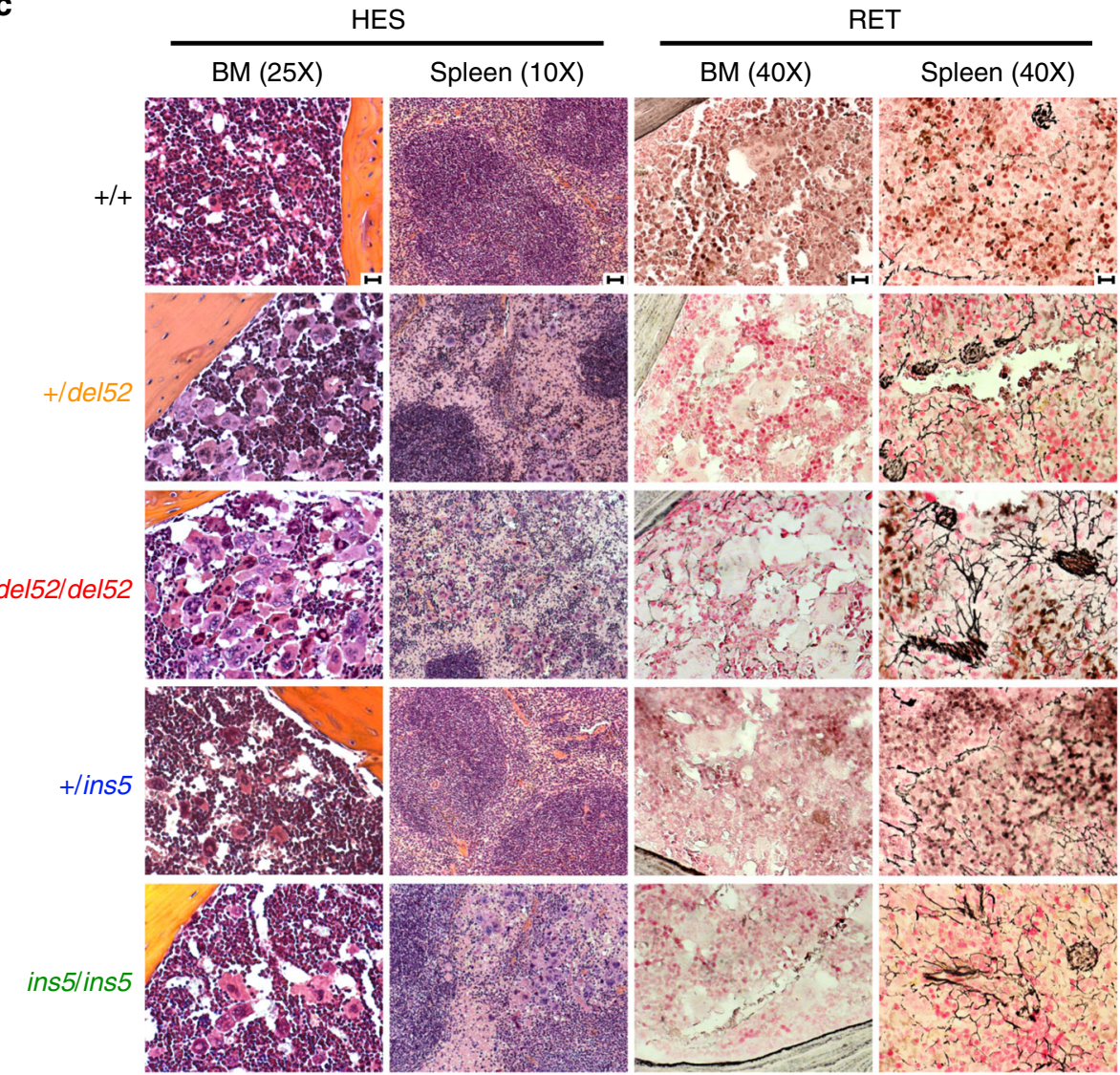

d Spleen del52/del52 HES (25X)

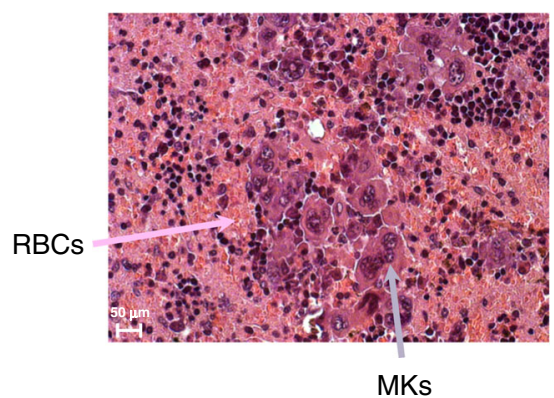

cells. Similarly, 4 months after induction of KI expression, HSCenriched BM cell populations $\left(\mathrm{Lin}^{-} \mathrm{Sca}-1^{+} \mathrm{C}-\mathrm{Kit}^{+} / \mathrm{LSK}\right.$ and SLAM) were $100 \%$ del52-mutated independently of the initial ratio engrafted while for initial ratios of $25 \%$ and $10 \%$ ins 5 BM cells, HSC populations still contained wt cells (Fig. 7c). More interestingly, the frequencies of SLAM in BM dose-dependently increased with higher initial amounts of transplanted del52/del52
BM cells whereas frequencies of ins5/ins5 SLAM remained independent of the initial percentages of engrafted mutated $\mathrm{BM}$ cells, suggesting a del52-induced increase in HSC symmetric division compared to ins5 (Fig. 7d). Taken together these results suggest that both del52 and ins 5 provide a competitive advantage to HSC but del52 induces a stronger amplification of the HSC compartment than ins5. 
Fig. 4 BM and spleen features of KI mice. a BM cellularity and $\mathbf{b}$ Spleen weight of $\geq 10$ months post-tamoxifen $\mathrm{KI}$ mice were expressed as means \pm SEM ( $n$ $=5$ different mice for each genotype). In the figure, white is used for $+/+$, orange for $+/$ del52, red for del52/del52, blue for $+/$ ins 5 and green for ins $5 /$ ins 5 genotypes. Significance was calculated with a two-sided parametric $t$-test. In $\mathbf{a},{ }^{\star} p=0.032(+/+$ vs. $+/$ del52), $0.0183(+/$ del52 vs. del52/del52), 0.0163 (del52/del52 vs. ins5/ins5); ${ }^{\star \star} p=0.036$ (del52/del52 vs. $+/$ ins5); ${ }^{\star \star \star} p=0.0005$ (+/+vs. del52/del52) and in b, ${ }^{\star} p=0.0126(+/+$ vs. ins5/ins5), 0.0238 ( + /ins5 vs. ins5/ins5); ${ }^{\star \star \star} p<0.0001$ except $p=0.0006$ for del52/del52 vs. ins5/ins5. Source data are provided as a Source data file. c Histopathology of $\mathrm{BM}$ and spleen showing HES and RET stainings of $\geq 10$ months post-tamoxifen mice. $\mathbf{d}$ HES staining showing MK hyperplasia and increase in red blood cells (RBCs) in spleen of homozygous del52/del52 mouse. Images were obtained using a DM2000 Leica microscope and a DFC300FX Leica camera with Leica Application Suite v.2.5,OR1 acquisition software $(\times 10, \times 25$, and $\times 40$ magnifications). Scale bars represent $50 \mu$ m. Similar results were obtained with analyzing at least three independent mice for each genotype.

Role of homozygosity in the expansion of CALR ins5 HSCs. On one hand, platelet counts were reported to be higher in ins5-ET than in del52-ET patients ${ }^{16,17}$ and, on the other hand, homozygous clones are more frequently found in ins5- than in del52mutated patients $2,3,18,19$. Thus, we decided to investigate how homozygous ins 5 cells might out-compete heterozygous ins 5 cells at the HSC level and how it would affect platelet levels knowing that thrombocytosis in homozygous ins5/ins 5 mice was significantly superior than in heterozygous $+/$ del52 mice (Fig. 8a). In order to test the effect of ins 5 homozygosity on the severity of the disease, we performed a competitive transplantation of $20 \%$ of ins5/ins 5 with $80 \%$ of + /ins 5 BM cells into lethally irradiated recipient mice. One month after transplantation, KI expression was induced by tamoxifen. As expected, mice engrafted with $100 \%$ heterozygous del52 cells displayed higher platelet counts than $100 \%$ heterozygous ins5-transplanted mice, compared to engraftment control with $100 \%$ wt $(+/+)$ cells (Fig. 8b). In the presence of limited homozygous ins 5 cells with heterozygous ins 5 cells, mice developed a significant stronger thrombocytosis than mice engrafted with $100 \%$ of heterozygous ins 5 cells, and surpassed, but not significantly, the $100 \%$ heterozygous del52 model. Six months after KI induction, the percentage of ins5/ins5 HSCs (SLAM) after single SLAM cell sorting and genotyping of colonies had doubled compared to initially engrafted BM cell levels (Fig. 8c) showing that homozygous ins5 cells can outcompete heterozygous ins 5 cells and may play an important role in the extent of thrombocytosis.

\section{Discussion}

We report here a direct comparison of the effects of physiological expression of the two most frequent CALR mutations, del52 and ins5, on hematopoiesis. Del52 KI mice developed a more severe thrombocytosis than ins $5 \mathrm{KI}$ mice and in the homozygous than in the heterozygous status. The majority of platelet increase in homozygous del52 KI mice seems to be due to an increase in both the number and the size of MKs, while homozygous ins5 and heterozygous del52 thrombocytosis was mostly due to an increase in MK size, confirmed by an increased ploidy.

The extent of thrombocytosis developed by KI mice was related to the zygosity status of the mutations, as observed for del52 by $\mathrm{Li}$ et al. ${ }^{13}$. The strategies used to generate the two KI models were different but result in the same chimera sequence (Supplementary Fig. 10). This suggests that the magnitude of thrombocytosis is dependent on the amount of CALR mutants and probably on the ratio of CALR mutant to CALR wt. Of note, CALR del52 and CALR ins5 proteins were barely detectable in heterozygous $\mathrm{KI}$ cells, may be due to the role of CALR wt in the stability of the mutants and/or increased secretion. It is interesting to highlight that with $100 \%$ mutated HSCs, CALR-mutated KI mouse models are more closely related to patients than $J A K 2^{\mathrm{V} 617 \mathrm{~F}} \mathrm{KI}$ mice because $C A L R$-mutated, in contrast to $J A K 2^{\mathrm{V} 617 \mathrm{~F}}$-patients, display a high allele burden (30-50\%) at the HSC/progenitor level suggesting that most HSCs need to be mutated for disease development ${ }^{20}$. In contrast to our KI and RV mouse models ${ }^{8}$, levels of platelets in ins5-ET patients were reported to be higher than in del52-ETs ${ }^{16,17}$. Several hypotheses may explain the apparent difference in the levels of del52- versus ins5-induced thrombocytosis in patients compared to mice. First, in agreement with the observations performed in mice, one study reported that $\mathrm{CD}_{3} 4^{+}$progenitors transduced to express del52 generated more proplatelets than with ins $5^{21}$. Second, in the mouse models, we can follow a time-dependent development of thrombocytosis, correlating with $\mathrm{HSC} / \mathrm{MkP}$ amplification. Platelet counts start decreasing when del52 mice display signs of MF-like features. In both mice and patients, CALR mutations induce a continuum of diseases between ET, pre-fibrosis, and PMF with fluctuating platelet levels. While ins5 ETs present slightly higher platelet counts than del52 ETs, this difference is not further observed in $\mathrm{MF}^{17}$. In particular, del52 ETs are more inclined to progress towards MF than ins5 ETs, thus suggesting more heterogeneity in platelet counts for del52 patients. Although allele burden in CALR-mutated patients often tends to be close to $50 \%$ when disease declare, it would be interesting to compare platelet levels in del52 and ins5 ETs with similar and low allele burden and to follow progression of thrombocytosis to measure the real impact of these two mutations on platelet counts. Third, it was reported that homozygous CALR mutations, although they are rare, were rather ins5 and type 2-like than del52 and type 1-like mutations $2,3,18,19$. In ET patients, homozygous ins 5 is frequently restricted to a small fraction of progenitors, but may contribute to high level of thrombocytosis. In favor of this hypothesis, when engrafting limited amount of homozygous ins 5 cells with heterozygous ins 5 cells in mice, the platelets counts undeniably exceeded levels reached by $100 \%$ heterozygous ins5-engrafted mice, and more interestingly, by $100 \%$ heterozygous del52engrafted mice. In this model, the homozygous ins 5 HSC reached $40 \%$ when thrombocytosis surpassed levels reached by the heterozygous del52 engrafted mice. In patients, the homozygous clone is lower, ranging from 2 to $25 \%$ in the CD34 ${ }^{+}$cell population, but it cannot be excluded that it is specifically amplified in the $\mathrm{MK} /$ platelet lineage. Thus, our study may provide a preliminary explanation on the apparent contrast between the del52 and ins5 thrombocytosis phenotype in mouse compared to human. Therefore, it will be important to correlate platelet counts with the percentages of homozygous CALR-mutated MKs or at least with the global $C A L R$-mutated allele burden in MKs.

In contrast to $C A L R$-mutated patients who show little leukocytosis compared to JAK2 $2^{\mathrm{V} 617 \mathrm{~F}} \mathrm{NMPs}$, we observed that del52 and, to a slightly lesser extent, ins5 KI mice develop leukocytosis that is dependent on zygosity. In a similar manner, Li J et al also showed a slight increase in WBC in heterozygous del52 KI mice, more reproducible and significantly higher in homozygous animals $^{13}$. Similarly to heterozygous del52 KI mice, there was a moderate leukocytosis in the CRISPR-Cas9-mediated murine del52 $\mathrm{KI}$ mice ${ }^{12}$, while no change in WBC counts was seen in the human del52 TG and the CRISPR-Cas9-mediated murine del61 and murine del19 $\mathrm{KI}$ mice ${ }^{10-12}$. We show here that the increased WBC population in del52 and ins 5 homozygous KI mice includes 
a

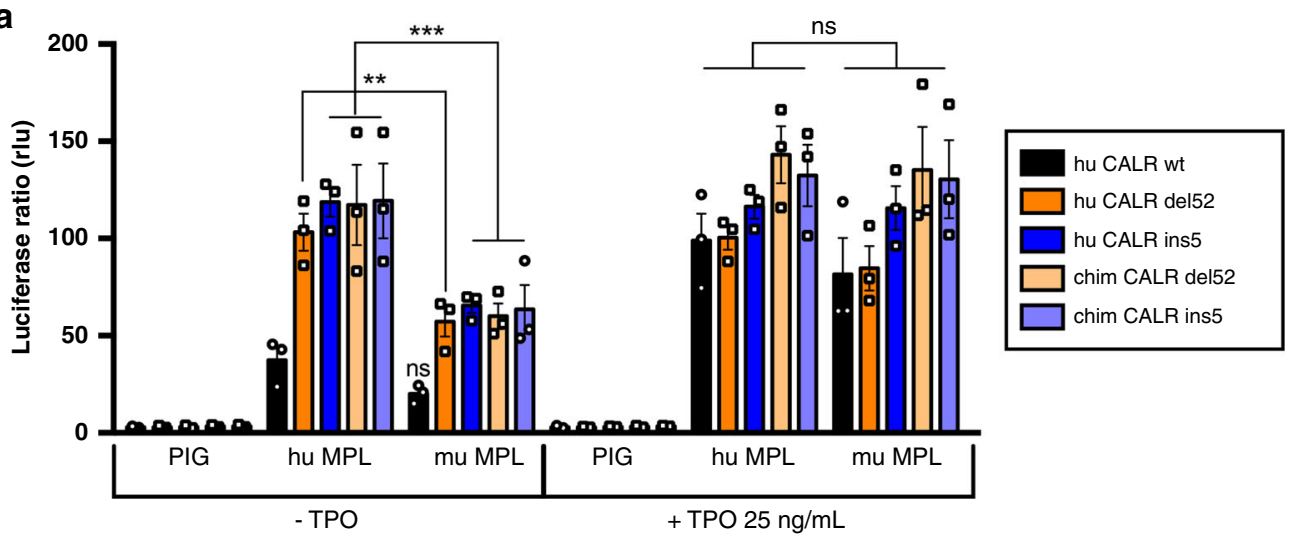

b
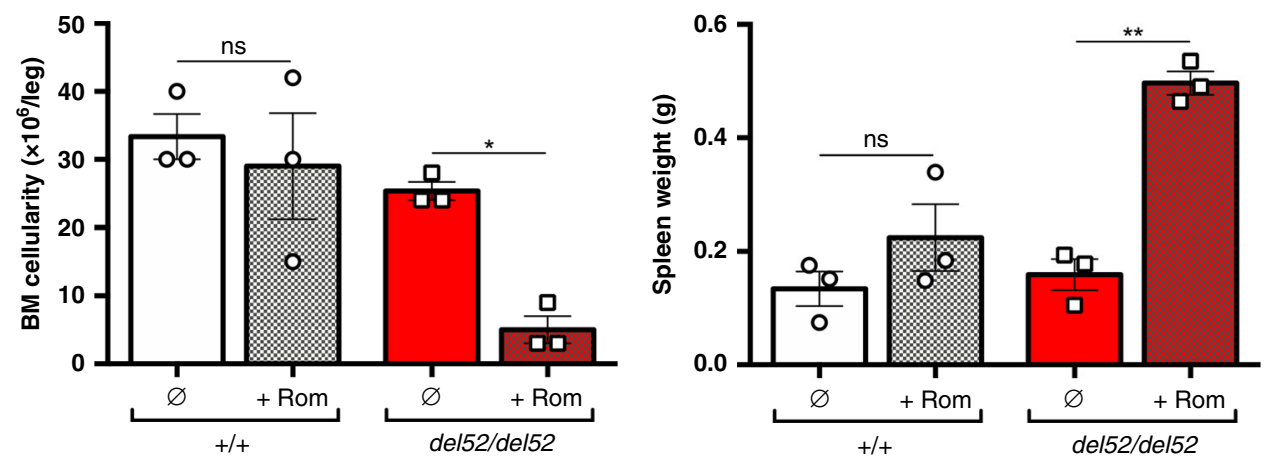

C

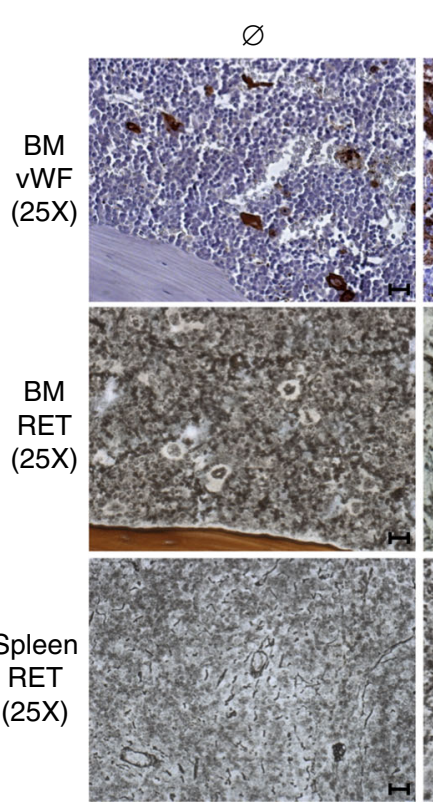

$+/+$

+ Rom del52/del52

$\varnothing$ del52/del52

+ Rom
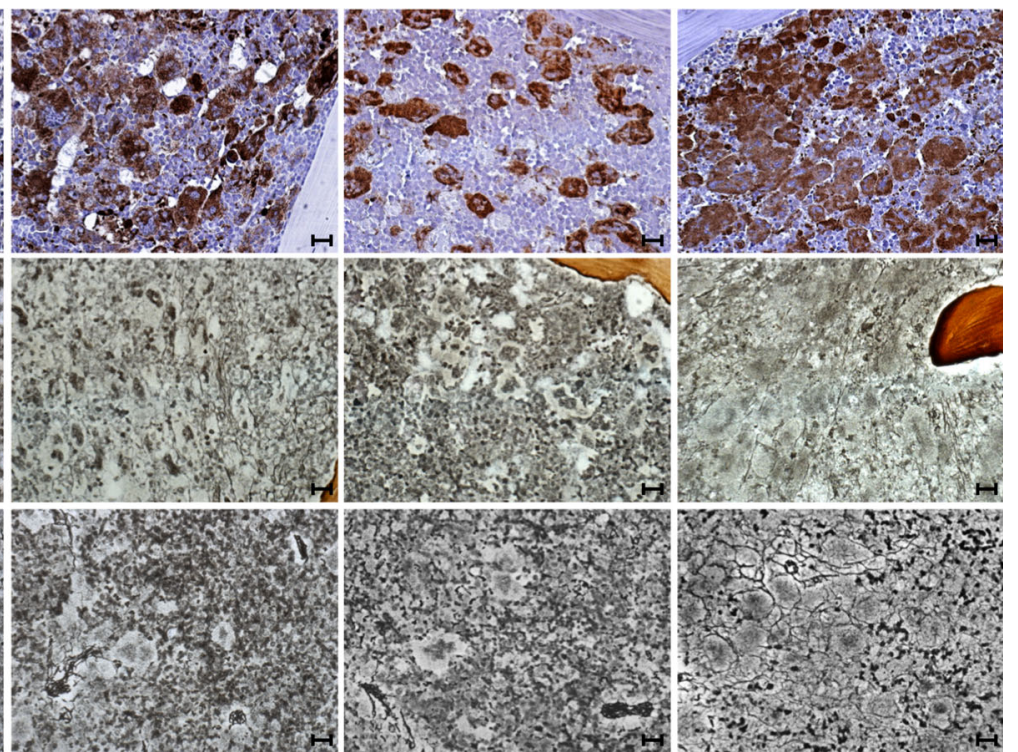

Fig. 5 Degree of activation of MPL/JAK2/STAT determines the phenotype. a The $\gamma 2 \mathrm{~A}$ cells were transfected to express JAK2, mu MPL, hu MPL or an empty vector (PIG) and equal amounts of either the hu CALR wt (black), del52 (dark orange) or ins5 (dark blue) or the chim CALR del52 (light orange) or ins5 (light blue), as indicated. STAT5-dependent transcriptional activity with or without TPO was measured $24 \mathrm{~h}$ after transfection by the dual firefly luciferase system with Spi-Luc reporter (STAT5 response elements) and renilla pRL-TK reporter with constitutive expression, as an internal control. Shown are means \pm SEM of three independent experiments in triplicate. Significance was assessed using Tukey's multiple comparison test $\left({ }^{\star \star} p=0.026\right.$; ${ }^{* \star \star} p=$ 0.0004 (hu CALR ins5), 0.0001 (chim CALR del52), 0.0002 (chim CALR ins5); ns not significant). b BM cellularity and spleen weight of 1 month posttamoxifen del52/del52 KI mice (red) and wild-type $(+/+)$ littermates (white) treated with romiplostim (+Rom, hatched histograms) or not $(\varnothing$, solid histograms). Data are means \pm SEM ( $n=3$ different mice) and significance of + Rom to $\varnothing$ condition was assessed using a two-sided parametric paired $t$-test: ${ }^{\star} p=0.0198 ;{ }^{\star \star} p=0.0017$; ns: not significant. Source data are provided as a Source data file. c BM and spleen were examined after vWF immunostainning and RET staining. Images were obtained using a DM2000 Leica microscope and a DFC300FX Leica camera with Leica Application Suite v.2.5,OR1 acquisition software ( $\times 25$ magnifications). Scale bars represent $50 \mu \mathrm{m}$. Similar results were obtained with analyzing three independent mice for each condition. 


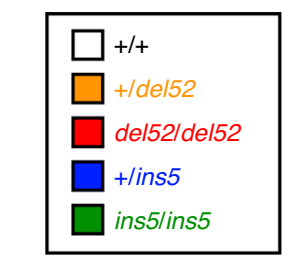

del52

b

\begin{tabular}{|c|c|}
\hline $\begin{array}{c}\text { del52/del52 } \\
\text { or ins5/ins5 } \\
\left(\mathrm{CD} 45.2^{+}\right)\end{array}$ & $\begin{array}{c}+/+ \\
\left(\mathrm{CD} 45.2^{+}\right. \\
\left.\mathrm{GFP}^{+}\right)\end{array}$ \\
\hline $100 \%$ & $0 \%$ \\
\hline $75 \%$ & $25 \%$ \\
\hline $50 \%$ & $50 \%$ \\
\hline $25 \%$ & $75 \%$ \\
\hline $10 \%$ & $90 \%$ \\
\hline $0 \%$ & $100 \%$ \\
\hline
\end{tabular}

\begin{tabular}{|c|c|}
\hline del52/del52 & ins5/ins5 \\
\hline (5\% & 引 $0 \%$ \\
\hline$\square 10 \%$ & $\square 10 \%$ \\
\hline$\square 20 \%$ & $\square 20 \%$ \\
\hline$\square 50 \%$ & $50 \%$ \\
\hline | 75\% & $75 \%$ \\
\hline $100 \%$ & $100 \%$ \\
\hline
\end{tabular}

a

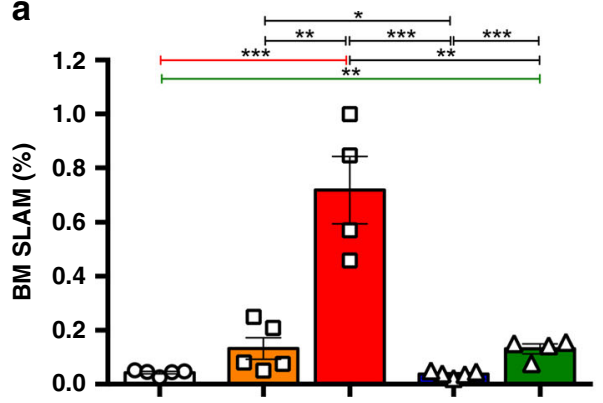

C

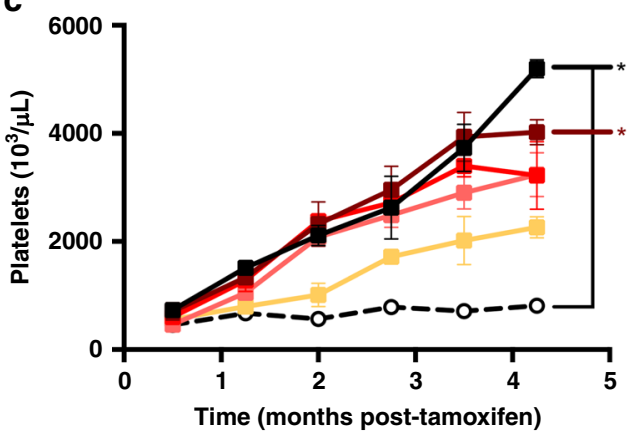

d

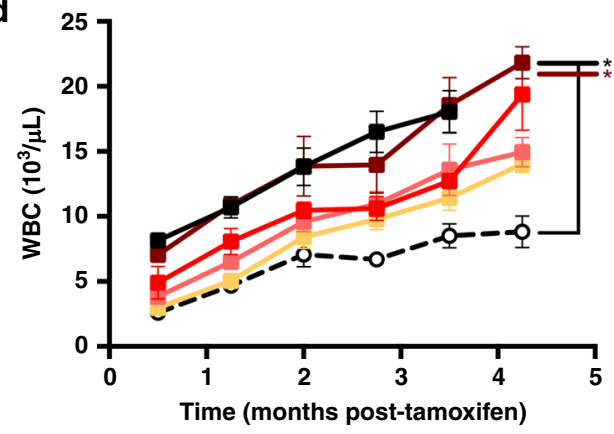

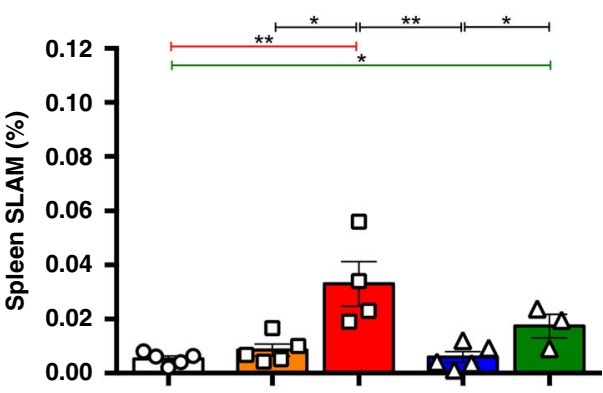
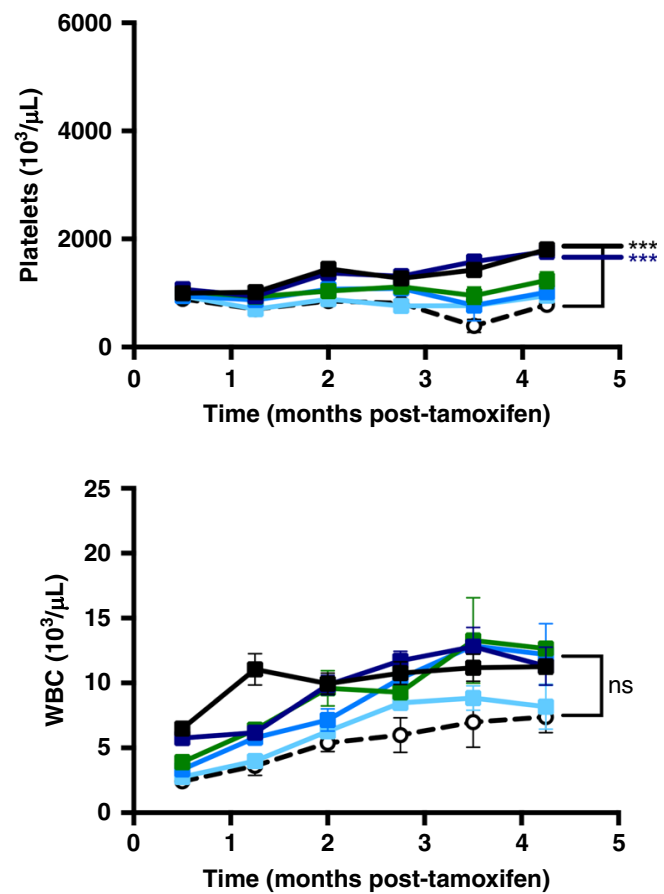

Fig. 6 Effect of CALR del52 and CALR ins 5 on the HSCs. a Percentages of HSCs (SLAM, Lin ${ }^{-}$c-Kit $^{+}$Sca- ${ }^{+}$CD150- CD48 ${ }^{+}$) in BM and spleen of $\geq 10$ months post-tamoxifen mice were expressed as means $\pm \operatorname{SEM}(n=5$ independent mice of $+/+$ (white), $+/$ del 52 (orange) and $+/$ ins 5 (blue) genotypes; $n=4$ del52/del52 (red) mice and for ins5/ins5 (green) genotype $n=4$ for BM and $n=3$ for spleen analyses). Significance was determined using a two-sided parametric $t$-test: in $\mathrm{BM},{ }^{\star} p=0.047 ;{ }^{\star \star} p=0.0012$ (+/+ vs. ins5/ins5), 0.0016 ( $+/$ del52 vs. del52/del52), 0.0034 (del52/del52 vs. ins5/ins5); ${ }^{\star \star \star} p=$ 0.0004 except 0.0009 for $+/$ ins 5 vs. ins $5 /$ ins 5 and in spleen, ${ }^{\star} p=0.0157$ ( $+/$ del52 vs. del52/del52), $0.0131\left(+/+\right.$ vs. ins5/ins5); ${ }^{\star *} p=0.0071(+/+$ vs. del52/del52), 0.0094 (del52/del52 vs. +/ins5), 0.0334 (+/ins5 vs. ins5/ins5). b To investigate the effect of CALR del52 and CALR ins5 on HSCs we engrafted different ratios of homozygous del52/del52 (yellow, pink, red, brown and black squares and solid lines) and ins5/ins5 (turquoise, blue, green, dark blue and black squares with solid lines) CD45.2+ KI BM cells with wild-type $(+/+)$ CD45.2+ GFP+ BM cells in CD45.1+ lethally irradiated mice. Four weeks after transplantation, $\mathrm{KI}$ allele expression was induced by tamoxifen and $\mathbf{c}$ platelet and $\mathbf{d}$ WBC counts were measured. Data are the means \pm SEM, del52/del52 platelets: $n=4,4,2,4,4,3$ for $0 \%, n=4,4,3,3,3,3$ for $10 \%, n=5,4,5,4,4,4$ for $25 \%, n=5,5,4,5,5,4$ for $50 \%, n=3,4,4,3,4,3$ for $75 \%$ and $n=5,5,5,3,5,2$ for $100 \%$; ins $5 /$ ins 5 platelets: $n=3,3,3,1,3,3$ for $0 \%, n=5,4,3,4,2,4$ for $10 \%, n=5,5,5,4,2,3$ for $25 \%, n=5,5,3,4,3,5$ for $50 \%, n=5,5,3,4,3,5$ for $75 \%$ and $n=5,5,3,4,4,5$ for 100\%; del52/del52 WBC: $n=4,4,3,4,4,4$ for $0 \%, n=4$ for $10 \%, n=5,4,5,5,4,5$ for $25 \%, n=5,5,5,5,5,4$ for $50 \%, n=4,4,4,3,4,4$ for $75 \%$ and $n=5,5,5,4,5$ for $100 \%$; ins 5 /ins 5 WBC: $n=3,3,3,2,3,3$ for $0 \%, n=5,5,4,4,5,5$ for $10 \%, n=5,5,5,5,3,4$ for $25 \%, n=5,5,5,5,3,5$ for $50 \%, n=5,5,3,5,4,5$ for $75 \%$ and $n=5,4,5,5,5,5$ for $100 \%$. N represents individual mice and varies depending on time of sampling during the course of the experiment and on the group defined as the percentage of engrafted homozygous cells, as indicated in the Source data file. Significance compared to the $0 \%$ condition (open circles with connecting black dashed line) was determined using ANOVA with Dunnett's multiple comparison test and a nonparametric Kruskal-Wallis's multiple comparison test for ins5/ins5 WBC. In $\mathbf{c}$, ${ }^{\star} p=0.0172$ (100\%), $0.0256(75 \%) ;{ }^{\star \star \star} p=0.0001(100 \%$ and $75 \%)$ and in $\mathbf{d},{ }^{*} p=0.0422(100 \%), 0.0128(75 \%)$; ns not significant. Source data are provided as a Source data file.

neutrophils, B and T lymphocytes, natural killer (NK) cells and eosinophils as well as monocytes and basophils that were differentially regulated by CALR del52 and ins5. The increase in neutrophils in del52 KI mice could be the consequence of activation of the G-CSF receptor by CALR mutants ${ }^{5}$. Moreover, as homozygous ins 5 patients were reported to present a deficiency in both the neutrophil myeloperoxidase (MPO) and the eosinophilic peroxidase $(\mathrm{EPX})^{22}$, it could be expected to see neutropenia in mice placed in a pathogen-challenged environment. Of particular interest, our KI models present high levels of circulating lymphocytes, especially of various sub-types of T cells. However, it is difficult to know whether this increase results from CALR mutant immunogenicity and the activation of lymphocytes. This hypothesis is a matter of debate as work from collaborators shows 

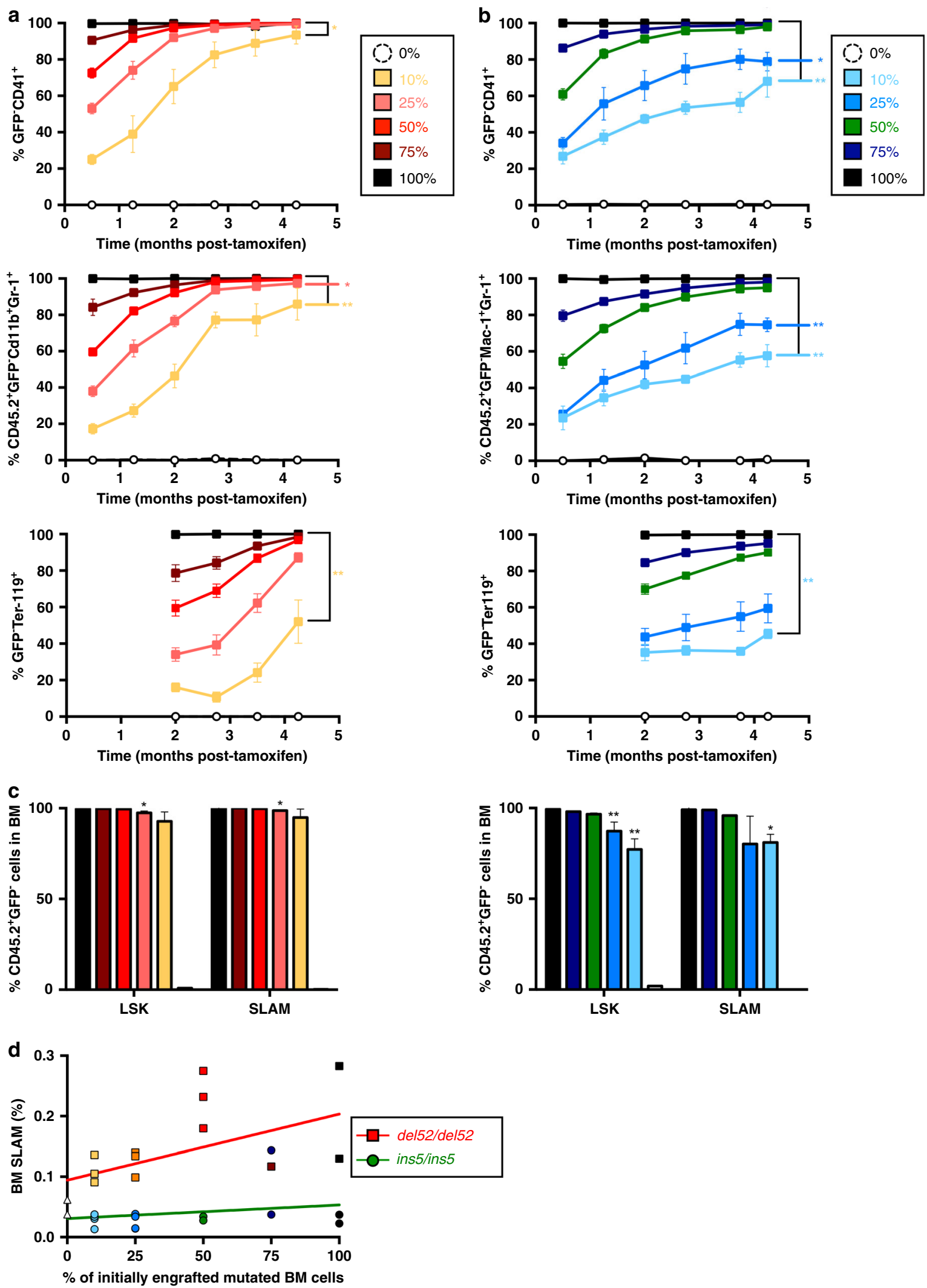

that CALR del52 has an immunosuppressive effect ${ }^{23}$ and causes a down-regulation of MHC-I endogenous antigen loading and presentation $^{24}$ but CALR mutant epitopes were also shown to stimulate $\mathrm{CD}^{+}$and $\mathrm{CD}^{+}{ }^{+} \mathrm{T}$ cells in patients ${ }^{25,26}$. Importantly, in contrast to $J A K 2^{\mathrm{V} 617 \mathrm{~F}}$, which is detected at a low level in lymphoid cells and rarely in T lymphocytes from MPN patients,
$C A L R$ mutations are present in the majority of monocytes and NK cells and frequently detected in T lymphocytes, most likely owing to the effect of both del52 and ins 5 on HSC expansion. Thus, the presence of CALR mutations in lymphoid cells might also have a direct effect on their expansion or their blood trafficking that should be investigated in the future. 
Fig. 7 Evolution of homozygous del52 and ins5 cells in blood and BM cells. Percentages of a del52/del52 (yellow, pink, red, brown and black squares and solid lines) and $\mathbf{b}$ ins5/ins5 (turquoise, blue, green, dark blue and black squares with solid lines) mutated KI cells in platelets (CD41 $\left.{ }^{+}\right)$and erythroid $\left(\right.$ Ter-119+) blood cells was determined as GFP- cells and in granulocytes (Cd11b+Gr-1+) as CD 45.2 ${ }^{+} \mathrm{GFP}-$ cells, and are expressed as means \pm SEM (in a: $n=4$ for $0 \%, n=4$ GFP-CD41+ $, n=4,4,4,2,4,4$ CD 45.2+GFP-Cd11b+Gr-1+ $n=4,4,4,3$ GFP-Ter-119+ for $10 \%, n=5$ for 25 and $50 \%, n=4,4,4,4$, $3,4 \mathrm{GFP}-\mathrm{CD}_{4} 1^{+}$and $n=4$ for $75 \%, n=5$ and 2 for the last point for $100 \%$; in $\mathbf{b}: n=3$ for $0 \%, n=4$ for $10 \%, n=4$ and 3 for the last point for $25 \%, n=5$ for $50 \%, 75 \%$, and $100 \%$, with $n$ being individual mice and varying depending on time of sampling during the course of the experiment and on the group defined as the percentage of engrafted homozygous cells, as indicated in the Source data file). Significance to the 100\% condition (black squares with connecting black solid line) was measured with nonparametric Kruskal-Wallis's multiple comparison test. In $\mathbf{a},{ }^{\star} p=0.0167(10 \%$, GFP-CD41+), 0.0334 $\left(25 \%, \mathrm{CD} 45.2^{+}{ }_{\mathrm{GFP}}-\mathrm{Cd} 11 \mathrm{~b}^{+} \mathrm{Gr}-1^{+}\right)$; ${ }^{\star \star} p=0.0023\left(10 \%, \mathrm{CD} 45.2^{+}{ }_{\mathrm{GFP}}-\mathrm{Cd} 11 \mathrm{~b}^{+} \mathrm{Gr}-{ }^{+}{ }^{+}\right), 0.0094(10 \%, \mathrm{GFP}-\mathrm{Ter}-119+)$ and in b, ${ }^{\star} p=0.0106(25 \%$, $\left.\mathrm{GFP}^{-} \mathrm{CD} 41^{+}\right) ;{ }^{\star \star} \mathrm{p}=0.0011\left(10 \%, \mathrm{GFP}^{-} \mathrm{CD} 41^{+}\right), 0.0098\left(25 \%, \mathrm{CD} 45.2^{+} \mathrm{GFP}^{-} \mathrm{Cd} 11 \mathrm{~b}^{+} \mathrm{Gr}-1^{+}\right), 0.0015\left(10 \%, \mathrm{CD}^{+} 5.2^{+} \mathrm{GFP}^{-} \mathrm{Cd} 11 \mathrm{~b}^{+} \mathrm{Gr}-1^{+}\right), 0.0081(10 \%$, GFP- $^{-}$- $-119^{+}$). c Percentages of homozygous del52 and ins 5 cells (CD45.2+ GFP- cells) were assessed in HSC-enriched BM LSK and SLAM cells and are shown as means \pm SEM, significance to the $100 \%$ condition with a two-sided parametric $t$-test: ${ }^{*} p=0.0344$ (25\% del52/del52, LSK), 0.0152 (del52/del52 SLAM), 0.0132 (10\%, ins5/ins5 SLAM); ${ }^{\star \star} p=0.0143$ (25\%, ins5/ins5 LSK), 0.0028 (10\%, ins5/ins5 LSK). d The percentages of BM SLAM 4 months after tamoxifen induction of $\mathrm{KI}$ expression were determined and expressed according to the percentages of initially engrafted homozygous del52 (colored squares and red solid line) and homozygous ins5 (colored circles and green solid line) BM cells. For $\mathbf{c}, \mathbf{d}, n=1-5$ independent mouse BM were analyzed, depending on the percentage of engrafted homozygous cells, as indicated in the Source data file. Source data are provided as a Source data file.
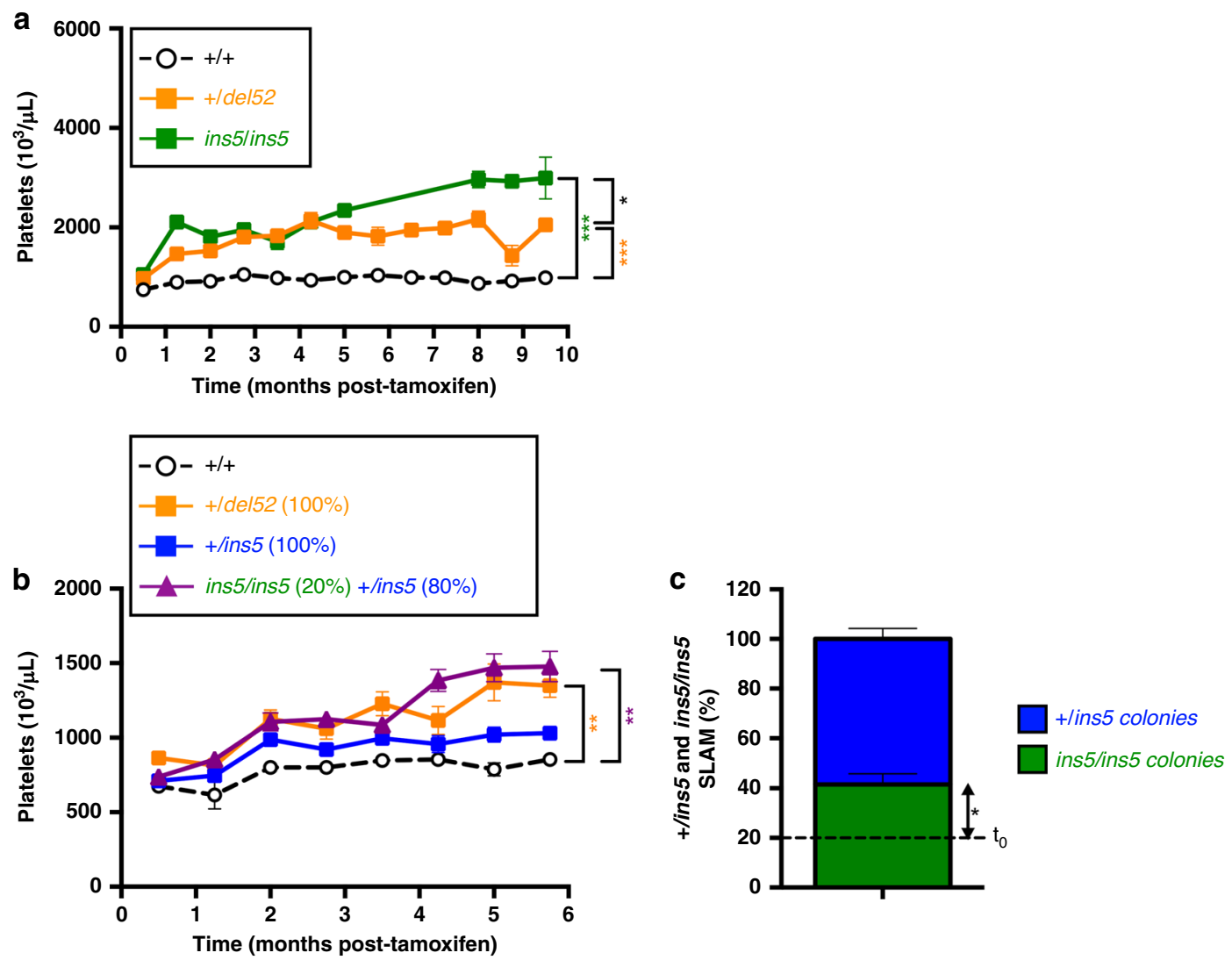

Fig. 8 Homozygous ins 5 cells drive the severity of the disease phenotype. a Comparison of the time-dependent increase in blood platelet levels of heterozygous $+/$ del 52 (orange squares and solid line) and homozygous ins5/ins 5 (green squares and solid line) $\mathrm{KI}$ mice after tamoxifen induction. Means $\pm \operatorname{SEM}(n=16,18,15,11,11,8,13,7,14,11,5,6,4+/+, n=18,20,15,12,10,7,11,7,12,12,8,5,10+/$ del52 and $n=6,1,6,8,9,7,5,6,5,5$ ins $5 /$ ins 5 independent mice, as shown in the Source data file) and significance was determined using ANOVA with Tukey's multiple comparison test ( ${ }^{\star} p=0.0365$; ${ }^{\star \star \star} p<0.001$ ). To investigate the effect of homozygous ins $5 /$ ins 5 cells in the development of thrombocytosis we engrafted $20 \%$ of ins5/ins5 $\mathrm{KI} \mathrm{BM}$ cells with $80 \%$ of heterozygous $+/$ ins $5 \mathrm{KI} \mathrm{BM}$ cells in lethally irradiated mice (purple triangles with solid line). As controls we also engrafted $100 \%$ wt ( $+/+$, open circles with black solid line), heterozygous $+/$ del52 orange squares with solid line) or $+/$ ins 5 (blue squares with solid line) KI BM cells. Four weeks after transplantation, $\mathrm{KI}$ allele expression was induced by tamoxifen and $\mathbf{b}$ platelets were measured. Figure shows the means \pm SEM $(n=6+/+n=8,8,8$, $8,7,8,8,8+/$ del $52, n=8$ and 7 for the last point $+/$ ins 5 and $n=8,8,8,8,3,8,7,7$ ins $5 /$ ins 5 and $+/$ ins 5 mixed individual mice, as indicated in the Source data file). Significance was calculated with a nonparametric Kruskal-Wallis's multiple comparison test $\left({ }^{\star *} p=0.0076(+/+v s .20 \%\right.$ ins $5 /$ ins 5$)$, 0.0083 (+/+ vs. 100\%.+/del52). c At 6 months post-induction, single BM SLAM were sorted for a 10-day culture with all cytokines. Colonies were genotyped to retrospectively identify percentages of ins $5 /$ ins 5 (green) and + /ins 5 (blue) SLAMs. Data are the means \pm SEM ( $n=3$ mice with an average of 58 genotyped colonies/mouse). Significance compared to the $20 \%$ of initially engrafted ins $5 /$ ins 5 BM cells (dashed line, $t_{0}$ ) was measured with a two-sided parametric $t$-test $\left({ }^{\star} p=0.0492\right)$. Source data are provided as a Source data file. 
Homozygous del52 and to a lesser extent homozygous ins 5 and heterozygous del52 led to the amplification of the HSC compartment in BM and spleen. Competitive BM engraftment of homozygous del52 and ins 5 cells confirmed that del52 activity is more potent than ins 5 in promoting an advantage over the wt hematopoiesis, as previously shown in the $\mathrm{RV}$ model ${ }^{8}$. Indeed, an increase in the percentage of ins5-mutated cells was very slow when initial engraftment was below $50 \%$ of mutated BM cells, especially in the $\mathrm{RBC}$ and granulocyte compartments but also in platelets and in the BM HSC compartment. As mentioned earlier, it was observed that homozygous clones are more often identified in ins5-patients than in del52-patients, suggesting the importance of a gene-dosage for ins 5 oncogenic activity. In contrast, an absence of growth advantage of del52 compared to CALR wt was observed using the del52 TG BM cells in an initial $50 \%$ competitive transplantation ${ }^{10}$. Similarly, the effect of del52 on HSC was absent when Li et al. performed a 50\% homozygous del52 $\mathrm{BM}$ cell competitive transplantation ${ }^{13}$. In these two studies, the heterozygous/homozygous del52 expression was induced in donor mice before engraftment while we engrafted wt cells and induced homozygous del52 KI expression 1 month later. The only difference between the models of the group of AR Green and of our laboratory is the type of transgenic mice that were crossed with the floxed KI mice, Mxl-Cre (ubiquitous expression), and SCL-CreER ${ }^{\mathrm{T}}$ (HSC and endothelial cell expression), respectively. Thus, our hypothesis is that either del52-expressing cells display a homing defect and/or that expression of del52 in the Mx1Cre context is not sufficient to induce a competitive advantage. Alternatively, it is also possible that the amplification of HSCs observed in KI mice may correspond to an increase of a subpopulation of active HSCs with more proliferating capacities, that would result in milder secondary engraftment. Interestingly, the group of K Shimoda showed that CALR wt is able to inhibit the CALR del52-induced competitive advantage, thus it is possible that the total absence of CALR wt in homozygous del52 or ins 5 HSCs might explain their advantage on their heterozygous counterparts ${ }^{27}$. With age, preferentially homozygous del52 KI mice developed splenomegaly accompanied with a decrease in BM cellularity, while blood parameters remained constant. A decrease in BM cellularity was partially explained with the striking MK hyperplasia and increase in MK ploidy, as well as the decrease in the erythroid lineage while the granulocytic lineage remained constant. Splenomegaly was due to extramedullary hematopoiesis characterized with an increased frequency of giant MKs, erythroid, and granulocytic cells at the expense of the lymphoid cell populations. There was no global decrease in RBC since the decrease in BM was compensated by splenic erythropoiesis. Surprisingly, while the role of MKs in the development of MF has been largely documented ${ }^{28}$, heterozygous del52 KI mice, like human del52 TG and murine del19, del52, and del61 KI mice, did not develop or only minor fibrosis even in aged animals, although they present increased numbers of MKs in both $\mathrm{BM}$ and spleen. The homozygous del52 $\mathrm{KI}$ mice, that presented an even higher MK hyperplasia in BM and spleen, showed minor fibrosis in BM and moderate fibrosis in the spleen. However, this difference in the grades of fibrosis severity seemed to be independent of different signaling (STAT5) intensities in BM versus spleen MKs. Conversely, moderate fibrosis in BM but not in the spleen was observed in the homozygous del52 $\mathrm{KI}$ mice of Li et al. while MK hyperplasia was observed in both tissues ${ }^{13}$. Moreover, the progression of homozygous ins5 $\mathrm{KI}$ mice to fibrosis was observed in rare cases with a lower grade compared to homozygous del52 KI mice, maybe due to the highest amplification of MKs in the del52 setting. These observations are coherent with the RV models where del52compared to ins5-expressing mice develop high-grade fibrosis in both BM and spleen from 6 months post-BM transplantation ${ }^{8}$. We hypothesized that the differences of fibrosis in the RV compared to the KI models could be due to the activation of mu MPL by hu
CALR mutants that were reported to be more efficient than the mouse/mouse combination ${ }^{11,12}$. As our KI mice express a murine CALR with a human mutated C-terminal tail chimeric protein, we tested to which extent they could activate mu MPL, compared to hu CALR del52 and ins5. The chimeric proteins were as efficient as hu CALR mutants to activate mu MPL, but chimeric protein and hu CALR were less active towards mu MPL than hu MPL. When MPL signaling in the homozygous del52 $\mathrm{KI}$ mice was stimulated by the TPO agonist romiplostim, fibrosis was visible in both BM and spleen at slightly higher levels than wt-treated littermates. Thus, the suboptimal activation of MPL by the chim CALR del52 and chim CALR ins5 in our KI mice, that was confirmed by the overactivation of STAT5 by in vitro TPO stimulation of MK might partially explain the mild fibrosis phenotype. Of note, we did not detect any significant difference between CALR del52 and CALR ins5-induced activation of STAT5 in $\gamma 2 \mathrm{~A}$ cell line. Whether this activation is also similar in vivo in HSCs or not and whether other signaling pathways may contribute to the distinct phenotypes developed by del52 and ins $5 \mathrm{KI}$ mice remain to be tested.

Overall, our KI mouse models show a differential effect of the two most frequent del52 and ins5 CALR mutations in mouse hematopoiesis, in both a heterozygous and a homozygous setting. Therefore, these KI mice provide a powerful tool to better understand the differences between del52 and ins5 CALR mutations and to perform preclinical studies. In addition, our observations suggest that homozygous ins 5 clone may play a more important role in the ET pathogenesis than previously thought, giving a future avenue of research in MPN pathogenesis.

\section{Methods \\ Generation of conditional heterozygous and homozygous CALR del52 and CALR ins5 KI mice. CALR del52 and ins5 KI mice were generated at the Institut} Clinique de la Souris (ICS, Illkirch, France). The targeting vectors were derived from a genomic DNA fragment of murine Calr gene obtained from 129S2/SvPas mouse genomic DNA library. The intron 8 of the gene was modified to integrate firstly a cassette containing a fused exon $8^{-}$exon 9 with the stop site and a triple polyA sequence surrounded with two LoxP sites, followed by a Pgk-NeoR cassette flanked by FRT sites and, secondly, a modified exon 9 with the 52-bp deletion (del52) or 5-bp insertion (ins5) mutation (untranslated). Due to the lack of homology between human and mouse in the alternative reading frame of the 3'UTR induced by the frameshift of the mutations, we chose an alternative strategy to express the putative del52 and ins 5 Calr-mutated murine genes by expressing a chimeric murine/human protein with most of the murine gene (except from the 52-bp deletion or 5-bp insertion in the last exon 9) and part of the $3^{\prime}$ UTR human $C A L R$, translated by the frameshift mutations. The human sequence was: AGGAT GATGAGGACAAAGATGAGGATGAGGAGGATGAGGAGGACAAGGAGGAA GATGAGGAGGAAGATGTCCCCGGCCAGGCCAAGGACGAGCTGTAGAGA GGCCTGCCTCCAGGGCTGGACTGAGGCCTGA, the human translated part after the mutated mouse CALR protein being: RRMMRTKMRMRRMRRTRRK MRRKMSPARPRTSCREACLQGWTEA.

The linearized homologous recombination vector constructs, including $\approx 4 \mathrm{~kb} 5^{\prime}$ and $3^{\prime}$ homology arms, were electroporated into C57BL/6N embryonic stem (ES) cells followed by G418 selection. Polymerase chain reaction (PCR) and Southern blot were used to screen and identify homologously recombined ES cells that were injected into blastocysts and reimplanted into the uterine horns of pseudopregnant foster females to allow embryos to develop to term. Chimeras were crossed with C57BL/6N Tac mice carrying the Flp recombinase transgene in order to excise the $P g k-N e o R$ cassette ${ }^{29}$. Agouti color hair progenies were genotyped for germinal transmission of the targeted allele using PCR and Southern blotting analyses. Subsequently, these LoxP KI mice were transferred, bred, and studied in the animal facilities of Gustave Roussy. Cre recombination after crossing the LoxP KI mice with Cre-expressing transgenic mice (HSC-SCL-Cre-ER ${ }^{\mathrm{T}}$ ) $^{30}$ results in excision of the fused normal exons and expression of the genes with the mutated exon 9. Three-month-old Cre/LoxP KI mouse progenies were treated with tamoxifen (200 mg/kg daily dose, Sigma-Aldrich) for 4 consecutive days.

Mouse genotyping. Conditional del52 KI mice were genotyped after genomic DNA extraction using the Mouse Direct PCR kit (Biotool) with a and b primers generating the wt (503 bp) and a conditional KI (404 bp) allele bands. The conditional ins5 KI mice genotyping was similarly performed by PCR with the primers $c$ and d amplifying the wt (217 bp) and the conditional KI (297 bp) alleles. The presence of the SCL-CreER ${ }^{\mathrm{T}}$ copies was detected by PCR with p1, p2, p3, and $\mathrm{p} 4$ generating a $700 \mathrm{bp}$ wt band (p1 and $\mathrm{p} 2)$ and a 1,100 bp SCL-CreER ${ }^{\mathrm{T}}$ band (p3 and 
p4). Primer sequences are listed in Supplementary Table 1. Gel imaging was analyzed with Image Lab software (Bio Rad).

Analysis of mice. Animal experiments were conducted in Gustave Roussy animal facility and approved by Gustave Roussy review board, protocol no. 2016-1047171. Mice were housed under 12-hour light/12-hour dark cycles and monitored ambient temperature $\left(21 \pm 1^{\circ} \mathrm{C}\right)$ and humidity range $(20-70 \%)$.

Blood, BM, and spleen. Platelet, hemoglobin, and WBC counts were determined using an automated counter (MS9; Schloessing Melet). BM cells were collected by flushing the femurs and tibias. Spleens were weighed and single-cell suspensions were prepared.

Content in progenitor and precursor cells by flow cytometry. Flow cytometry (LSRFortessa, FACSCanto X, BD Biosciences) with BD FACSDiva software was used to determine the cell content of BM and spleen with appropriate Abs. Lin cells were selected on the absence of labeling with APC-conjugated rat Abs against Ter-119, B220, Gr-1, CD3, and CD11b. Lin $^{-}$Sca- $1^{+}$c-Kit ${ }^{+}$(LSK) population was stained with Abs against Sca-1 (PE-Cy7) and c-Kit (PerCP-Cy5.5) and the LSKCD48-CD150+ (SLAM) population enriched in HSCs was isolated with antiCD48 (Pacific Blue) and CD150 (BV510) Abs. Anti-CD41 (FITC) Ab was used to label MK progenitor $(\mathrm{MkP})$ cells with the $\mathrm{LKSCa}-1^{-} \mathrm{CD} 150^{+} \mathrm{CD} 41^{+}$combination. $\mathrm{BM}$ and spleen precursors were labeled as $\mathrm{CD} 41^{+} \mathrm{CD} 42^{+}(\mathrm{MK}), \mathrm{Cd} 11 \mathrm{~b}^{+} \mathrm{Gr}-1^{+}$ (granulocyte lineage), $\mathrm{CD}^{+}$and $\mathrm{B}^{+} 20^{+}(\mathrm{T}$ and $\mathrm{B}$ cells) and Ter-119+ (erythroid cells). All Abs were obtained from BioLegend (Ozyme). Data were analyzed with Kaluza Analysis software (Beckman Coulter).

Analysis of WBC populations by flow cytometry. To investigate the cell population composition of WBCs (gating strategy in Supplementary Fig. 2a), we labeled blood using anti-Ly6G (APC-Cy7) Ab to identify polynuclear neutrophils (Ly6G ${ }^{+}$). In the $\mathrm{Ly}_{6 \mathrm{G}}{ }^{-}$cell population we labeled B and T lymphocytes as B220+ (BV510) and $\mathrm{CD}^{+}(\mathrm{PB})$, respectively. Among the $\mathrm{Ly}^{+} \mathrm{G}^{-} \mathrm{B} 220^{-} \mathrm{CD} 3^{-}$cells, NK cells were stained as $\mathrm{NKp}^{+} 6^{+}$(FITC) while monocytes were stained as $\mathrm{CD}_{11 b^{+}}(\mathrm{PE})$ $\mathrm{Ly}^{-1+}$ (APC) in the $\mathrm{Ly}^{-1+} \mathrm{G}^{-} \mathrm{B} 220^{-} \mathrm{CD}^{-}{ }^{-} \mathrm{NKp} 46^{-}$cell population. Gating on $\mathrm{Ly}_{6 \mathrm{G}^{-}} \mathrm{B} 220^{-} \mathrm{CD} 3^{-} \mathrm{NKp} 46^{-} \mathrm{CD}^{-} 1 \mathrm{~b}^{-}$cells we used anti-CD45 (PE-Texas Red) $\mathrm{Ab}$ and evaluated granularity with the sidewards scatter (SSC) parameter to distinguish eosinophils (CD45 high SSC ${ }^{\text {high }}$ ) from basophils (CD45 ${ }^{\text {lows }} S^{\text {low }}$ ). We further investigated different lymphocyte sub-populations (gating strategy in Supplementary Fig. $3 \mathrm{a}$ ) in the $\mathrm{Ly}_{6} \mathrm{G}^{-}$cell population by combining Abs to detect T-cytotoxic lymphocytes as $\mathrm{CD}^{+}$(Pacific Blue) $\mathrm{CD}^{-}$(APC) $\mathrm{CD}^{+}(\mathrm{PE})$ and T-helper lymphocytes $(\mathrm{TH})$ as $\mathrm{CD}^{+}{ }^{+} \mathrm{CD} 4^{+} \mathrm{CD}^{-}$. In the $\mathrm{TH}$ gate, we used anti-FoxP3 (Alexa Fluor 488) and anti-CD25 (BV510) Abs to stain regulatory CD4 ${ }^{+} \mathrm{T}$ cells (Tregs, $\mathrm{CD}^{+}{ }^{+} \mathrm{CD} 4{ }^{+} \mathrm{CD} 8^{-}$FoxP3 $^{+}{ }^{+} \mathrm{CD} 25^{+/-}$) and conventional CD4 ${ }^{+} \mathrm{T}$ cells (TC, $\mathrm{CD}^{+}{ }^{+} \mathrm{CD} 4{ }^{+} \mathrm{CD} 8{ }^{-}$FoxP3 ${ }^{-} \mathrm{CD} 25^{-}$). All Abs and the FoxP3 Fix/Perm buffer set were from BioLegend. Data were acquired with BD FACSDiva software and analyzed with Kaluza Analysis software (Beckman Coulter).

Progenitor assays in semi-solid cultures. Spleen and BM cells were plated in methylcellulose MethoCult 32/34 (Stemcell Technologies) with $10 \mathrm{ng} / \mathrm{mL}$ hu TPO (a generous gift from Kirin, Tokyo, Japan), $1 \mathrm{U} / \mathrm{mL}$ hu EPO (PreproTech), $10 \mathrm{ng} /$ $\mathrm{mL} \mathrm{mu}$ IL-3 (Miltenyi Biotec), $10 \mathrm{ng} / \mathrm{mL}$ hu IL-6 (Miltenyi Biotec) and $100 \mathrm{ng} / \mathrm{mL}$ mu SCF (PreproTech). After 7 days, colonies derived from erythroid (BFU-E, burst forming unit-erythroid) and granulo-monocytic (CFU-GM, colony forming unitgranulocyte macrophage) progenitors were counted. For megakaryocytic progenitor (CFU-MK) assay, BM nucleated cells were plated in duplicate in serum-free fibrin clot assays with $25 \mathrm{ng} / \mathrm{mL}$ mu SCF, $25 \mathrm{ng} / \mathrm{mL}$ hu IL-6 and without or with 20 $\mathrm{ng} / \mathrm{mL}$ hu TPO. CFU-MKs were evaluated at day 7 by acetylcholinesterase staining.

Histology of BM and spleen. Tibia and spleen sections were fixed in $4 \%$ paraf ormaldehyde, decalcified, and paraffin-embedded. Sections were stained with HES for cytology and MKs were immune-stained with rabbit anti-vWF Ab. Reticulin fibers were revealed by Gordon and Sweet's silver staining.

Romiplostim treatment. One month after tamoxifen induction of KI allele expression, homozygous del52/del52 KI mice and wt $(+/+)$ littermates were administered $1 \mathrm{mg} / \mathrm{kg}$ romiplostim (Nplate, Amgen) by subcutaneous injection on days 1,8 , and 15 based on a published study ${ }^{15}$. Platelets, WBC and hemoglobin were recorded on days 1 and 15 . At day 20 mice were sacrificed to determine BM cellularity, spleen weight and the presence of reticulin fibers (Gordon and Sweet's silver staining) and MKs (vWF immunohistochemical staining) in BM and spleen.

Ploidy of BM MKs. BM cells were labeled with FITC-conjugated anti-CD41 and APC-conjugated anti-CD42 Abs (BioLegend). After hypotonic cell permeabilization with $0.1 \%$ sodium citrate, DNA content was stained with $50 \mu \mathrm{g} / \mathrm{mL}$ propidium iodide (Sigma) and treated with $50 \mu \mathrm{g} / \mathrm{mL}$ RNAase (Sigma). Ploidy was measured by flow cytometry in the $\mathrm{CD} 41^{+} \mathrm{CD} 42^{+}$cell population by means of a LSRFortessa
(Becton Dickinson) with a BD FACSDiva software. Data were analyzed with Kaluza Analysis software (Beckman Coulter). The mean ploidy was calculated in $\geq 8 \mathrm{~N}$ MKs $((8 \mathrm{~N} \times$ number of cells at $8 \mathrm{~N}$ ploidy level $+\ldots+64 \mathrm{~N} \times$ number of cells at $64 \mathrm{~N}$ ploidy level)/total number of cells).

Western blot analysis. $\mathrm{Lin}^{-}$cells isolated from BM were analyzed by western blot to determine the expression levels of CALR using a rabbit monoclonal Ab directed against the N-terminal portion of CALR (Cell Signaling, Ozyme), common to wt as well as mutant CALR. An Ab directed against amino acids 50-150 of human CALR from Abcam was also used, as well as a mouse monoclonal Ab specifically directed against the C-neoterminus of mutant CALR (Dianova). Mouse monoclonal anti- $\beta$ Actin (Sigma) served to monitor the loading. Blot was quantified using ImageJ software (National Institute of Health).

Dual luciferase transcriptional assay. The KI allele encodes the mouse CALR ins5 or del52 with human mutated C-terminal tail. These chimeric CALR del52 and CALR ins 5 cDNAs were amplified by PCR from homozygous del52 and ins 5 KI BM cells and introduced into a pMSCV-IRES-GFP vector. Transcriptional activation of STAT5 was analyzed in $\gamma 2 \mathrm{~A}$ cells using the dual firefly reporter Spi-Luc and pRLTK-driven renilla luciferase system (Promega). $\gamma 2 \mathrm{~A}$ cells were transiently transfected using Lipofectamine 2000 reagent (ThermoFisher) with cDNAs coding for mu STAT5, mu JAK2 as the cells are defective in JAK2, hu CALR wt, hu CALR del52, hu CALR ins5, chim CALR del52 or chim CALR ins5 in the presence of mu MPL, hu MPL, or the empty pMX-IRES-GFP (PIG) vector, as indicated. Cells were stimulated or not with $25 \mathrm{ng} / \mathrm{mL}$ TPO (Miltenyi Biotec) for $24 \mathrm{~h}$.

Phosphoflow assay. $\mathrm{Lin}^{-}$cells isolated on ice from BM and spleen from $\geq 10$ months post-tamoxifen del52/del52 KI mice and from wt $(+/+)$ littermates were stimulated or not with $100 \mathrm{ng} / \mathrm{mL}$ TPO for $15 \mathrm{~min}$ or incubated with $1 \mu \mathrm{M}$ ruxolitinib (Selleck Chemicals, Euromedex) for $1 \mathrm{~h}$ at $37^{\circ} \mathrm{C}$, as indicated. Cells were fixed with $1.6 \%$ formaldehyde (ThermoFisher) and MKs were stained with Abs against CD41 (Alexa Fluor 700) and CD42d (APC) from BioLegend. After methanol permeabilization and staining for p-STAT5 (BV421, BD Biosciences), samples were analyzed by flow cytometry (LSRFortessa) with BD FACSDiva software. Data were analyzed with Kaluza Analysis software (Beckman Coulter).

Competitive transplantation models. All procedures were approved by the Gustave Roussy Ethics Committee (protocol 2016-104-7171). Lethally irradiated CD $45.1^{+}$wt recipient mice (C57BL/6LY5.1, Charles River) were engrafted with $3.10^{6}$ of different ratios $(100 \%, 75 \% ; \% ; 25 \% ; 10$ and $0 \%)$ of BM cells from CD45.2+ floxed homozygous del52 or ins5 KI mice with transgenic CD45.2 $2^{+}$wt mice expressing GFP (UBI-GFP/BL6) ${ }^{31}$. Four weeks after transplantation, KI allele expression was induced by tamoxifen. Blood parameters were followed every 3 weeks and the competitive advantage of the mutated del52/del52 or ins5/ins 5 cells in the blood was monitored by flow cytometry as $\mathrm{GFP}^{-} \mathrm{CD} 41^{+}$in platelets, $\mathrm{GFP}^{-}$Ter- $119^{+}$in erythrocytes and as $\mathrm{CD} 45.2^{+} \mathrm{GFP}^{-} \mathrm{Cd} 11 \mathrm{~b}^{+} \mathrm{Gr}-1^{+}$in granulocytes. At 4 months after induction, the percentages of mutated cells were analyzed in LSK/SLAM compartments of both BM and spleen.

A competitive ins $5 \mathrm{KI}$ transplantation model was made by engrafting $3.10^{6}$ of a limited number of floxed homozygous ins5/ins5 KI BM cells (20\%) in presence of floxed heterozygous + /ins 5 KI cells $(80 \%)$ into lethally irradiated wt recipient mice (C57BL/6JRj, Janvier Labs). As controls, $100 \%$ of floxed $+/$ ins $5 \mathrm{KI},+/$ del52 KI, and $+/+\mathrm{BM}$ cells were also engrafted, as indicated. After 4 weeks, KI allele expression was induced by tamoxifen and blood parameters were analyzed every 3 weeks. At 6 months post-induction, SLAM cells were labeled from Lin ${ }^{-}$cells isolated from $\mathrm{BM}$ and sorted (Influx, Becton Dickinson) at 1 cell per well in a 96-well plate. After 10 days of culture in a serum-free medium supplemented with $10 \mathrm{ng} / \mathrm{mL}$ hu TPO, $1 \mathrm{U} / \mathrm{mL}$ hu EPO, $20 \mathrm{ng} / \mathrm{mL}$ hu G-GSF (a generous gift from Gustave Roussy Hospital), $10 \mathrm{ng} / \mathrm{mL} \mathrm{mu}$ IL-3, $10 \mathrm{ng} / \mathrm{mL}$ hu IL-6, $100 \mathrm{ng} / \mathrm{mL} \mathrm{mu}$ SCF, and $100 \mathrm{ng} /$ $\mathrm{mL}$ hu FLT-3L (Miltenyi Biotec), colonies were lysed to recover genomic DNA for PCR genotyping to retrospectively identify heterozygous and homozygous ins5 KI SLAM (Supplementary Fig. 11). Primers used to detect the wt mouse Calr allele (302 bp) were muCALR_wt_ex8_F and muCALR_wt_onT2_R. Primers used to detect the ins5 KI allele (177 bp) were muCALR_wt_int8-9_F and chimCALR_ins5_R. All primer sequences are listed in Supplementary Table 1.

Statistical analysis. Statistical analyses were performed using the Prism software. If not otherwise mentioned, tests used an unpaired design. Comparisons were made using a parametric two-tailed $t$-test or a nonparametric Mann-Whitney test when needed, and ANOVA with Tukey's or Dunnett's multiple comparison tests or nonparametric Kruskal-Wallis multiple comparison test, as indicated. Differences were significative for $p<0.05$.

\section{Data availability}

The authors declare that all relevant data supporting the findings of this study are included in the paper and its supplementary information files. Source data are provided with this paper. 
Received: 15 November 2019; Accepted: 4 September 2020;

Published online: 28 September 2020

\section{References}

1. James, C. et al. A unique clonal JAK2 mutation leading to constitutive signalling causes polycythaemia vera. Nature 434, 1144-1148 (2005).

2. Nangalia, J. et al. Somatic CALR mutations in myeloproliferative neoplasms with nonmutated JAK2. N. Engl. J. Med. 369, 2391-2405 (2013).

3. Klampfl, T. et al. Somatic mutations of calreticulin in myeloproliferative neoplasms. N. Engl. J. Med. 369, 2379-2390 (2013).

4. Pikman, Y. et al. MPLW515L is a novel somatic activating mutation in myelofibrosis with myeloid metaplasia. PLoS Med. 3, e270 (2006).

5. Chachoua, I. et al. Thrombopoietin receptor activation by myeloproliferative neoplasm associated calreticulin mutants. Blood 127, 1325-1335 (2016).

6. Elf, S. et al. Mutant calreticulin requires both its mutant c-terminus and the thrombopoietin receptor for oncogenic transformation. Cancer Discov. 6, 368-381 (2016).

7. Cabagnols, X. et al. Differential association of calreticulin type 1 and type 2 mutations with myelofibrosis and essential thrombocytemia: relevance for disease evolution. Leukemia 29, 249-252 (2015).

8. Marty, C. et al. Calreticulin mutants in mice induce an MPL-dependent thrombocytosis with frequent progression to myelofibrosis. Blood 127, 1317-1324 (2016).

9. Toppaldoddi, K. R. et al. Rare type 1-like and type 2-like calreticulin mutants induce similar myeloproliferative neoplasms as prevalent type 1 and 2 mutants in mice. Oncogene 38, 1651-1660 (2019).

10. Shide, K. et al. Calreticulin mutant mice develop essential thrombocythemia that is ameliorated by the JAK inhibitor ruxolitinib. Leukemia 31, 1136-1144 (2017).

11. Shide, K. et al. Mice with Calr mutations homologous to human CALR mutations only exhibit mild thrombocytosis. Blood Cancer J. 9, 42 (2019).

12. Balligand, T. et al. Knock-in of murine Calr del52 induces essential thrombocythemia with slow-rising dominance in mice and reveals key role of Calr exon 9 in cardiac development. Leukemia 34, 510-521 (2020).

13. Li, J. et al. Mutant calreticulin knockin mice develop thrombocytosis and myelofibrosis without a stem cell self-renewal advantage. Blood 131, 649-661 (2018).

14. Balligand, T. et al. Pathologic activation of thrombopoietin receptor and JAK2-STAT5 pathway by frameshift mutants of mouse calreticulin. Leukemia 30, 1775-1778 (2016).

15. Maekawa, T. et al. Myeloproliferative leukemia protein activation directly induces fibrocyte differentiation to cause myelofibrosis. Leukemia 31, 2709-2716 (2017).

16. Tefferi, A. et al. Type 1 versus Type 2 calreticulin mutations in essential thrombocythemia: a collaborative study of 1027 patients. Am. J. Hematol. 89, E121-E124 (2014).

17. Pietra, D. et al. Differential clinical effects of different mutation subtypes in CALR-mutant myeloproliferative neoplasms. Leukemia 30, 431-438 (2016).

18. Stengel, A. et al. Detection and characterization of homozygosity of mutated CALR by copy neutral loss of heterozygosity in myeloproliferative neoplasms among cases with high CALR mutation loads or with progressive disease. Haematologica 104, e187-e190 (2019).

19. El-Khoury, M. et al. Different impact of calreticulin mutations on human hematopoiesis in myeloproliferative neoplasms. Oncogene 39, 5323-5337 (2020).

20. Rumi, E. et al. JAK2 or CALR mutation status defines subtypes of essential thrombocythemia with substantially different clinical course and outcomes. Blood 123, 1544-1551 (2014).

21. Kollmann, K. et al. A novel signalling screen demonstrates that CALR mutations activate essential MAPK signalling and facilitate megakaryocyte differentiation. Leukemia 31, 934-944 (2017).

22. Theocharides, A. P. A. et al. Homozygous calreticulin mutations in patient with myelofibrosis lead to acquired myeloperoxidase deficiency. Blood 127, 3253-3259 (2016).

23. Liu, P. et al. Immunosuppression by mutated calreticulin released from malignant cells. Mol. Cell 77, 748-760.e9 (2020).

24. Arshad, N. \& Cresswell, P. Tumor-associated calreticulin variants functionally compromise the peptide loading complex and impair its recruitment of MHCI. J. Biol. Chem. 293, 9555-9569 (2018).

25. Holmström, M. O. et al. The calreticulin (CALR) exon 9 mutations are promising targets for cancer immune therapy. Leukemia 32, 429-437 (2018).
26. Cimen Bozkus, C. et al. Immune checkpoint blockade enhances shared neoantigen-induced T-cell immunity directed against mutated calreticulin in myeloproliferative neoplasms. Cancer Discov. 9, 1192-1207 (2019).

27. Shide, K. et al. Calreticulin haploinsufficiency augments stem cell activity and is required for onset of myeloproliferative neoplasms. Blood 136, 106-118 (2020).

28. Wagner-Ballon, O. et al. Monocyte/macrophage dysfunctions do not impair the promotion of myelofibrosis by high levels of thrombopoietin. J. Immunol. 176, 6425-6433 (2006).

29. Birling, M.-C., Dierich, A., Jacquot, S., Hérault, Y. \& Pavlovic, G. Highlyefficient, fluorescent, locus directed cre and FlpO deleter mice on a pure C57BL/6N genetic background. Genesis 50, 482-489 (2012).

30. Göthert, J. R. et al. In vivo fate-tracing studies using the Scl stem cell enhancer: embryonic hematopoietic stem cells significantly contribute to adult hematopoiesis. Blood 105, 2724-2732 (2005).

31. Schaefer, B. C., Schaefer, M. L., Kappler, J. W., Marrack, P. \& Kedl, R. M. Observation of antigen-dependent CD8+ T-cell/ dendritic cell interactions in vivo. Cell. Immunol. 214, 110-122 (2001).

\section{Acknowledgements}

This work was supported by grants from Ligue Nationale Contre le Cancer ("Equipe labellisée 2018", HR); Institut National du Cancer (PLBIO-2015, -2017 and -2018, IP), MPN Research Foundation (IP and JLV) and Institut National de la Santé et de la Recherche Médicale (Inserm). The "Investissements d'avenir" program is funding the Labex GR-Ex (IP, WV). MCC was funded by São Paulo Research Foundation 2016/ 03265-3. We thank O. Bawa for histology, the staff of the animal facilities of Gustave Roussy and C. Catelain and Y. Lecluse at the Imaging and Cytometry Platform. We thank M-C. Birling and E. Geronimus at the Institut Clinique de la Souris (Illkirch) for the generation of KI mice.

\section{Author contributions}

C.M., I.P., and W.V. conceived and designed the study, interpreted the data and wrote the paper. C.M., C.B., and M.C.C. designed and performed experiments, analyzed the data and prepared the figures. A.N. performed and analyzed luciferase experiments and V.E. treated mice by romiplostim and analyzed data. D.M., L.T., and PG contributed to the bone marrow transplantation and mouse study and PR to flow cytometry experiments and analysis. S.N.C., H.R., and J.L.V. designed experiments analyzed the data and wrote the paper.

\section{Competing interests}

The authors declare no competing interests.

\section{Additional information}

Supplementary information is available for this paper at https://doi.org/10.1038/s41467 020-18691-3.

Correspondence and requests for materials should be addressed to C.M.

Peer review information Nature Communications thanks the anonymous reviewer(s) for their contribution to the peer review of this work. Peer reviewer reports are available.

Reprints and permission information is available at http://www.nature.com/reprints

Publisher's note Springer Nature remains neutral with regard to jurisdictional claims in published maps and institutional affiliations.

Open Access This article is licensed under a Creative Commons Attribution 4.0 International License, which permits use, sharing, adaptation, distribution and reproduction in any medium or format, as long as you give appropriate credit to the original author(s) and the source, provide a link to the Creative Commons license, and indicate if changes were made. The images or other third party material in this article are included in the article's Creative Commons license, unless indicated otherwise in a credit line to the material. If material is not included in the article's Creative Commons license and your intended use is not permitted by statutory regulation or exceeds the permitted use, you will need to obtain permission directly from the copyright holder. To view a copy of this license, visit http://creativecommons.org/ licenses/by/4.0/.

(C) The Author(s) 2020 